\title{
Performance Analysis of Multichannel Medium Access Control Algorithms for Opportunistic Spectrum Access
}

\author{
Przemysław Pawełczak, Student Member, IEEE, Sofie Pollin, Member, IEEE, Hoi-Sheung Wilson So, Member, IEEE, \\ Ahmad R. S. Bahai, Member, IEEE, R. Venkatesha Prasad, Member, IEEE, and Ramin Hekmat
}

\begin{abstract}
In this paper, different control channel (CC) implementations for multichannel medium access control (MAC) algorithms are compared and analyzed in the context of opportunistic spectrum access (OSA) as a function of spectrum-sensing performance and licensed user activity. The analysis is based on a discrete Markov chain model of a subset of representative multichannel OSA MAC classes that incorporates physical layer effects, such as spectrum sensing and fading. The analysis is complemented with extensive simulations. The major observations are given as follows: 1) When the $\mathrm{CC}$ is implemented through a dedicated channel, sharing such dedicated channel with the licensed user does not significantly decrease the throughput achieved by the OSA network when the data packet sizes are sufficiently large or the number of considered data channels is small. 2) Hopping OSA MACs, where the $\mathrm{CC}$ is spread over all channels, are less susceptible to licensed user activity than those with a dedicated CC (in terms of both average utilization and on/off times). 3) Scanning efficiency has a large impact on the achievable performance of licensed and OSA users for all analyzed protocols. 4) The multiple rendezvous MAC class, which has yet to be proposed in OSA literature, outperforms all the multichannel MAC designs analyzed in this paper.
\end{abstract}

Manuscript received March 20, 2008; revised August 26, 2008. First published November 17, 2008; current version published May 29, 2009. This work was supported by the Dutch Ministry of Foreign Affairs under the Freeband AAF Project. The work of S. Pollin was supported by the Marie Curie OIF Fellowship of the European Union. This paper was presented in part at the 2008 IEEE Global Communications Conference, New Orleans, LA, November 30December 4, 2008, and The Third International Conference on Cognitive Radio Oriented Wireless Networks and Communications, Singapore, May 15-17, 2008. The review of this paper was coordinated by Dr. E. Hossain.

P. Pawełczak and R. V. Prasad are with the Department of Electrical Engineering, Mathematics, and Computer Science, Delft University of Technology, 2600 GA Delft, The Netherlands (e-mail: p.pawelczak@tudelft.nl; vprasad@tudelft.nl).

S. Pollin is with the Department of Electrical Engineering and Computer Science, University of California, Berkeley, CA 94720-4-1770 USA, and also with the Interuniversity Microelectronics Center, 3001 Leuven, Belgium (e-mail: pollins@eecs.berkeley.edu).

H.-S. W. So was with the Department of Electrical Engineering and Computer Science, University of California, Berkeley, CA 94720-4-1770 USA. He is now with the Siemens Technology-to-Business Center, Berkeley, CA 94704 USA (e-mail: so@eecs.berkeley.edu).

A. R. S. Bahai is with the Department of Electrical Engineering and Computer Science, University of California, Berkeley, CA 94720-4-1770 USA, with the Department of Electrical Engineering, Stanford University, Stanford, CA 94305-9515 USA, and also with National Semiconductor, Santa Clara, CA 95052-8090 USA (e-mail: bahai@eecs.berkeley.edu).

R. Hekmat is with the Department of Electrical Engineering, Mathematics, and Computer Science, Delft University of Technology, 2600 GA Delft, The Netherlands, and also with Capgemini Nederland B.V., 3528 BJ Utrecht, The Netherlands (e-mail: r.hekmat@tudelft.nl).

Digital Object Identifier 10.1109/TVT.2008.2009350
Index Terms-Communication channels, communication systems, multiaccess communication, radio spectrum management, resource management.

\section{INTRODUCTION}

$\mathbf{S}$ TATIC spectrum assignment, which has been applied to radio frequencies for almost a century, has led to a quasiscarcity of the spectrum. Therefore, finding a new unassigned frequency slot has forced system designers toward higher frequencies, e.g., $60 \mathrm{GHz}$. However, most of the already allocated radio bands are not used or are sporadically used. Then, it is logical to allow unlicensed users to exploit these frequencies when they are free at a specific place and time. Theoretically, such approach will increase the overall frequency reuse and boost the throughput for applications that opportunistically use the empty frequencies. This communication technique is called opportunistic spectrum access (OSA). More discussion on OSA and its relation to cognitive radio can be found in [1]-[4].

The concept of OSA, with it being very promising and attractive, introduces many new research challenges. The main research questions are related to preserving the quality of service (QoS) for primary users (PUs) while offering a fair level of service to secondary users (SUs). To protect the PU's QoS, the SUs need to accurately detect the presence of the PUs; however, they are prone to errors due to propagation conditions and noise. Whenever the SU nodes make a wrong observation about channel availability, their transmissions will harm the operation of the PU. Considering the QoS of the SU, it is clear that the QoS is largely determined by the channel utilization intensity and traffic pattern of the PUs [1]. The question arises as to whether it is acceptable for the PU that the SU opportunistically claims its channels (because the SU can never guarantee to the PU the avoidance of harmful interference since any detection process is prone to errors). However, there are many examples where it makes sense to minimally reduce the QoS of the PU if this would result in a much larger QoS improvement for the SU. Consider, for instance, a licensed user who is interested in periodically broadcasting traffic information. First, such broadcast is not substantially demanding in terms of data throughput; therefore, an opportunistic user can take advantage of the idle periods between broadcasts. Second, for a car interested in traffic information, missing a single broadcast is not that harmful, and a considerable amount of data 
traffic collisions could, hence, be tolerated. With OSA applied to this scenario, both the SUs and PUs can easily share the available spectrum while enjoying sufficient QoS.

The two phenomena strictly related to OSA, i.e., PU detection errors and channel availability variation, have a strong impact on the medium access control (MAC) protocol design for OSA networks, and the goal of this paper is to study the performance of different MAC classes in the context of OSA. OSA network architectures can be classified into centralized and distributed. In the centralized architecture, one dedicated network entity is responsible for the management of the medium access and functionalities related to channel management. Examples of these architectures for OSA are Common Spectrum Coordination Channel [5], Spectrum Information Channel [6], and DIMSUMNet [7]. For the distributed case, each node of the network locally decides on the channel access. The focus of this paper is the analysis of distributed OSA MACs only since centralized solutions are, in our opinion, not suitable for OSA networks and cognitive networks in particular, which, by definition, are distributed and self-organized.

There are many distributed OSA MAC designs that are already available in the literature, and since the most important feature of OSA is channel agility, the majority of the proposed protocols operate in a multichannel way. In this paper, we give an extensive overview of the already available OSA MAC protocols, identifying all the important features that are distinct to the OSA operation and necessary to be modeled. So far, none of the studies has focused on comparing these proposals and on how multichannel MAC design and PU detection quality impact the OSA network performance. We note that the only papers that focus on assessing the performance of spectrum sharing in general are [8] and [9]. However, they neglect many implementation details.

Our starting point for the analysis is the multichannel MAC comparison framework presented in [10], which was extended to the context of OSA. A detailed physical layer model with fading, PU activity, PU detection, and packet capture is added. Using this model, we show that a control channel (CC) solely dedicated to the OSA network is not needed since PU activity on such a channel does not significantly degrade the OSA throughput if the OSA data packets are large enough or the number of OSA channels is small. We also show that the throughput achieved in the OSA network linearly scales with the PU activity. However, depending on how efficiently the MAC design allows one to claim a free spectrum, the slope of the decrease in throughput as a function of PU activity is different. We also show that, for any OSA MAC, there is an optimal point between throughput loss caused by collisions with PU, false alarms, and sensing overhead.

The remainder of this paper is organized as follows: Section II introduces a characterization of different OSA MAC designs proposed in the literature. Section III introduces the analytical model and discusses the numerical results for the throughput assessment of distributed OSA multichannel MAC designs, particularly from the physical layer perspective. Simulation results for delay, throughput, and interference to the PU are explained in Section IV. Finally, this paper concludes in Section V.
TABLE I

SuRVEY OF Distributed OSA MACS With RESPECT TO THEIR Features (SEe Also [12, TAB. 1] AND [1, TAB. 2])

\begin{tabular}{c|c|c|c|c}
\hline Protocol & Bootstrapping & Type & Scan. & RFEs \\
\hline \hline DOSS [13] & Yes & DCC & Yes & 3 \\
Su et. al. [14] & No & DCC & Yes & 2 \\
Motamedi et. al. $[15]$ & No & DCC & Yes & 2 \\
SCA-MAC [16] & No & DCC & Yes & 2 \\
Nan t. al. [17] & No & DCC & Yes & 2 \\
C-MAC [18] & Yes & DCC & Yes & 1 \\
AS-MAC [19] & Yes & DCC & Yes & 1 \\
ESCAPE [20] & No & DCC & Yes & 1 \\
MMAC-CR [21] & No & DCC & Yes & 1 \\
DC MAC [22] & No & DCC & Yes & 1 \\
HC-MAC [23] & No & DCC & Yes & 1 \\
Choi et. al. [24] & No & DCC & No & 2 \\
Shu et. al. $[25]$ & No & DCC & No & 2 \\
BB-OSA [26] & No & DCC & No & 1 \\
OS-MAC [27] & No & DCC & No & 1 \\
HD-MAC [28] & Yes & SPCC & Yes & 1 \\
SRAC [29] & No & SPCC & No & 1
\end{tabular}

\section{OSA MAC CLASSIFICATION}

In Table I, we have listed different OSA MAC designs found in the literature. ${ }^{1}$ The identified protocol features that are important in the context of OSA are described in detail in the succeeding sections. More discussion on the identified features can be found in [1] and [12].

\section{A. Bootstrapping and Channel Selection}

Bootstrapping is the process during which an SU node decides which PU channels are suited for opportunistic use. In one scenario, external entities provide information on opportunistically enabled channels. Then, the SU consults those entities when it wants to join a network. Other scenarios assume that each node locally finds those channels, which can involve a significant amount of spectrum scanning. After finding the channels, each node distributes its set of channels to other users in the network, e.g., via a proprietary protocol. Interestingly, only a handful of proposed OSA MACs consider bootstrapping, e.g., C-MAC [18], AS-MAC [19], DOSS [13] (only for a CC), and HD-MAC [28]. Bootstrapping, which is sometimes also referred to as channel selection [30], is mostly explored in a single-channel context. In the multichannel domain, MAC designers usually assume that each OSA node has a preprogrammed list of PU channels to use. We will also assume in our analysis and simulations that the list of channels is known to the SU.

\section{B. CC Design}

After the bootstrapping procedure, the SU network decides on a set of possible channels. Now, for each data packet transmission, the SU transmitter and receiver have to coordinate which channel and time slot they will use for the

\footnotetext{
${ }^{1}$ We are aware of the existence of MAC for IEEE 802.22 WRAN [11] and proprietary OSA MACs found in the OSA devices of Shared Spectrum Company, Philips, and Microsoft, but since their specifications are not public, they are not mentioned here.
} 
transmission. This coordination is typically implemented with a (common) $C C$. From a reliability viewpoint, this $\mathrm{CC}$ is a very crucial element of the MAC design since no SU data communication is possible when it is obstructed.

Using the approach defined in [10, Sec. II] for general multichannel MACs, we can identify four classes of CC implementation. The list of references for each MAC class in the non-OSA case is presented in [10].

1) Dedicated (common) $C C$ (DCC), where one channel is solely dedicated to the transport of control messages. All nodes should overhear the control message exchange, even during data exchanges. As a result, one radio frontend (RFE) needs to be dedicated to the exchange of control messages. When only one RFE is used, the transmission of control and data packets is time multiplexed, but then, the operation of the protocol becomes more complex. The drawback of the DCC approach in the context of OSA is that, when a PU is active on the CC, all SU communications must wait until the dedicated channel becomes free again. If the PU activity is organized in long bursts, long delays may occur. As a result, existing OSA MAC protocols often assume that the $\mathrm{CC}$ is always free from the PU. Interestingly, the majority of the proposed designs so far are DCC (see Table I).

2) Hopping $C C$ (HCC), where all idle nodes hop across all channels, following the same pattern. When both the sender and the receiver successfully exchange control messages on the current channel, they stop hopping and start transmitting data. After that, they come back to the original hopping pattern, rejoining the rest of the network. HCC has the advantage of using all channels for transmission and control, whereas in DCC, the CC can be used to transfer only control packets. In addition, $\mathrm{HCC}$ does not require a single channel to be free from PU activity. This MAC class has not been proposed yet in the context of OSA in the literature. We note that, in HCC, only one control message can be exchanged during one hopping sequence.

3) Split phase CC (SPCC), where time is divided into alternating control and data phases. During a control phase, all nodes switch their RFEs to the dedicated CC and decide on the channels to use for data transfers in the upcoming phase. Each node stays on the negotiated channel during the data transmission phase. The advantage is that the $\mathrm{CC}$ can be used during the data phases. Compared to DCC, no extra RFE for the CC is needed. On the other hand, SPCC requires stronger synchronization to identify control and data phases. Only two out of all the identified MAC protocols in Table I belong to the SPCC class.

4) Multiple rendezvous CC (MRCC), where multiple nodes can exchange control information at the same time using all available channels. Each node knows the hopping patterns of its one-hop neighbors. Such hopping pattern is based on the seed of a pseudorandom generator. Once the seed of the receiver is known, the sender can follow the intended receiver on its hopping sequence. MRCC randomly spreads both control and data exchanges across all channels. As a result, $\mathrm{MRCC}$ is the most robust to unpredictable PU activities. We will quantitatively illustrate this later in this paper. However, MRCC also requires a more stringent synchronization between the hopping users since they have to keep track of the hopping times of their one-hop neighbors. In contrast to HCC, it allows one to exchange more than one message during the control sequence. Interestingly, none of the MAC designs in OSA context proposed so far belongs to the MRCC class of multichannel MAC protocols. A detailed discussion on the operation of the MRCC MAC proposal for a non-OSA environment can be found in [31].

We can further classify the multichannel MACs into two major groups: 1) with dedicated $C C$, i.e., DCC and SPCC, and 2) with hopping, i.e., HCC and MRCC. The multichannel MAC analysis framework of [10] will be used to compare these MAC designs in the context of OSA in Sections III and IV-B. Graphical representation of each multichannel OSA MAC class can be found in [1, Fig. 2].

\section{PU Detection}

Since an SU must not use a channel when a PU is present, it should obtain information about PU activities. Typically, this is implemented using PU detectors [32]. Alternatively, PU activity information can be assumed to be broadcasted by a central device. We can thus classify OSA MAC protocols into scanning and nonscanning OSAs. From Table I, we can conclude that the majority of the considered protocols assume having the scanning under their control.

Unfortunately, scanning increases the overhead since nodes cannot transmit when they are scanning. The overhead can be minimized by increasing the hardware complexity, i.e., by introducing a dedicated RFE for scanning only (see Table I for the list of OSA MACs and the corresponding number of RFEs). More importantly, since it is often difficult to distinguish SU and PU signals, the whole SU network has to be quiet during sensing, which requires quiet period management [18]. Scanning, or hence quieting the network, can periodically be done during or before each transmission attempt. The time interval between two consecutive sensing attempts varies and is often a function of the policy. The more tolerant the PU is to interference, the less often the sensing should be done. Noise, fading, multipath shadowing, and low PU signal levels make the reliable detection process difficult. Suboptimal detectors affect not only the PU QoS levels but also the SU QoS levels. In Section III-B1, we will give a detailed model of the PU detector, which will later be used in the global system model for the MAC comparison.

\section{Number of RFEs}

Different multichannel MAC designs operate using different numbers of RFEs. For DCC, this number varies between one (one RFE is time shared for control and data exchange) and three (one RFE for control data exchange and two simplex RFEs for data transfer). All the SPCC MACs proposed use only 
one RFE (see Table I). We expect that HCC and MRCC will use only one RFE as well.

The number of RFEs used by the node has a significant impact on the hardware cost, as well as the power consumption. The energy consumption of a radio depends, to a large extent, on the amount of time the RFE can be switched off to sleeping mode. Since none of the considered classes of MACs has been optimized for sleeping, we can assume that they cannot be turned off. As a result, the number of RFEs is roughly proportional to the total power consumption. Thus, in the remainder of this paper, we will not study the power cost in more detail and will assume that the number of RFEs is two for DCC and one for the other protocols.

\section{ANALYSIS}

In this section, we propose a model for analyzing OSA MAC performance from the throughput perspective. We start from the multichannel MAC model proposed in [10], which we extend to include OSA features such as PU channel occupation and an improved physical layer for capturing interference (from PU and SU) and PU detection performance. First, we give a global overview of the system model. Then, we discuss the detailed physical layer model. Finally, we embed them into the multichannel MAC model proposed in [10].

We note that our model possesses the same limitations as those in [10]. Specifically, due to the significant involvement of the delay analysis, the model is limited to throughput assessment only. In addition, this work is limited to a single collision domain, and effects such as hidden/exposed terminals are not modeled. However, features that are not captured by this analytical model, e.g., delay and level of interference to the PU, will be simulated in Section IV.

\section{A. Modeling Assumptions}

This section introduces simplifying assumptions that will allow us to capture the throughput of the considered MAC protocols in an intuitive analytical model. The parameters of the introduced model are summarized in Table II.

1) Channel Organization: We evaluate the performance of an SU network consisting of $N$ nodes that operates on a pool of $M_{D}$ channels, where each channel can potentially be used by a PU independent with probability $q_{p}$. Each channel has a fixed throughput of $C \mathrm{Mb} / \mathrm{s}$. The SUs can utilize the channels when they are temporarily not used by the PU but only when this causes no excessive interference to the PU of any channel $m \in M_{D}$.

2) Time Granularity: The system is analyzed using discrete time slots. As a result, all transmissions within the SU network are slotted, and the boundaries between consecutive slots are uniquely identified. In addition, for the analysis, the slots of the secondary network are assumed to be synchronized with those of the PU network. This means that any PU or SU packet starts at a slot boundary. A slot is long enough to contain one request-to-send (RTS)/clear-to-send (CTS) exchange of the SU network. In [1, Fig. 2], a more detailed illustration is given since SU transmissions are not synchronized with the PU slot, and a PU packet can start in between the RTS and CTS exchange.
TABLE II

SUMMARY OF PARAMETERS, GROUPED (From TOP TO BOTTOM) INTO MAC PARAmETERs, PHYSICAL PARAMETERs, AND ANALYTICAL MOdel PARAMETERS

\begin{tabular}{|c|c|c|}
\hline Parameter & Description & Unit \\
\hline$C$ & Average channel throughput & $\mathrm{b} / \mathrm{s}$ \\
\hline$l$ & Slot length & $\mu \mathrm{s}$ \\
\hline$m$ & Individual PU channel & - \\
\hline$M_{C}$ & Number of effective data channels & - \\
\hline$M_{D}$ & Number of channels & - \\
\hline$N$ & Number of SU nodes & - \\
\hline$p$ & Probability of data packet generation & - \\
\hline$p_{s}$ & Probability of successful arrangement (sim.) & - \\
\hline$q^{-1}$ & SU average data packet length & slots \\
\hline$q_{p}$ & Probability of channel occupancy by PU per slot & - \\
\hline$R$ & System throughput & $\mathrm{b} / \mathrm{s}$ \\
\hline$R_{o p t}$ & Optimized system throughput & $\mathrm{b} / \mathrm{s}$ \\
\hline$s$ & Scanning length & $\mu \mathrm{s}$ \\
\hline$t_{p}$ & Channel switching time & $\mu \mathrm{s}$ \\
\hline$h_{\max }$ & Maximum number of sensing samples & 一 \\
\hline$n$ & Number of interferers & - \\
\hline$N_{0}$ & Noise power & $\mathrm{dBm}$ \\
\hline$p_{d}$ & Probability of detection & - \\
\hline$p_{f}$ & Probability of false alarm & - \\
\hline$P_{P}$ & Power of PU interference & $\mathrm{dBm}$ \\
\hline$P_{S}$ & Power of SU packet & $\mathrm{dBm}$ \\
\hline$S_{x, n}(\varphi)$ & Capture probability & - \\
\hline$W$ & Channel bandwidth & $\mathrm{Hz}$ \\
\hline$\alpha$ & Interference constraint & - \\
\hline$\gamma$ & Instantaneous SNR for the energy detector & $\mathrm{dB}$ \\
\hline $\bar{\gamma}$ & Average SNR of the energy detector & $\mathrm{dB}$ \\
\hline$\varphi$ & Packet capture theshold & $\mathrm{dB}$ \\
\hline$\theta$ & PU signal detection threshold & $\mathrm{dB}$ \\
\hline$j$ & Number of successes in arrangement/termination & - \\
\hline$k$ & Number of communicating pairs & - \\
\hline $\mathbf{P}$ & Transition probability matrix & - \\
\hline$p_{k, z}$ & Transition probability from state $k$ to $z$ & - \\
\hline$S_{j}^{(\dot{k})}$ & Probability of successful arrangement & - \\
\hline$T_{j}^{(k)}$ & Probability of successful termination & - \\
\hline$\psi()$. & Slot availability & - \\
\hline$\rho$ & Channel utilization & - \\
\hline
\end{tabular}

3) Channel Access: Every node has an infinite stream of data to transmit. Distributed access ${ }^{2}$ is modeled by generating each channel access with probability $p$. Such channel access results in a connection arrangement (RTS/CTS exchange) on the CC. A successful arrangement is always made when only one user requests a new connection in the network in a given slot and the slot is free from PU activity. In addition, in case of collisions with the PU or another SU, the SU nodes are potentially able to capture the transmitted packet under the condition that the total signal-to-interference-plus-noise ratio is larger than a certain threshold $\varphi$.

4) Data Model: After obtaining the channel, the SU nodes can transmit only one packet (amount of data), which is fragmented into multiple slots, with an acknowledgement (ACK) per fragment. When the transmission has finished, the nodes need to perform a new arrangement for the next transmission attempt. For simplicity of analysis, the packet lengths of the secondary network are geometrically distributed with parameter $q$, i.e., each SU data packet has a mean length of $q^{-1}$ time slots.

\footnotetext{
${ }^{2}$ We note, just as in [10], that effects related to PU activity and multiplechannel management are much more profound for the operation of any multichannel OSA MAC than collision-resolution strategies. Therefore, the collision-resolution procedure, i.e., RTS/CTS exchange, assumed in the model is simplified.
} 
5) PU Detection: To avoid excessive interference with the PU, every slot of length $l$ starts with scanning period $s$. At the beginning of scanning period $s$, all the SU nodes stop their transmission, and at the end of such period, each secondary node locally decides if the channel is free from PU activity or not. Every decision is prone to errors. When an SU node does not detect a PU, with probability $1-p_{d}$, a collision between the $\mathrm{PU}$ and SU happens. A capture model determines if the collision will result in a lost packet for the SU. A lost fragment has to be retransmitted in the next available slot. Alternatively, an idle slot may be considered busy with false alarm probability $p_{f}$. In such a case, the $\mathrm{SU}$ postpones its transmission and tries to send the remainder of the packet in the next available slot. In both cases, when this happens during the data transmission phase, no new arrangement is needed. As a result, the presence of the PU only extends the data phase. Probabilities $p_{f}$ and $p_{d}$ are functions of $s$, channel bandwidth $W$, and detection threshold $\theta$. We also assume that all the OSA nodes observe the same channel conditions, i.e., the same instantaneous signal level from PU, in each slot.

6) Physical Layer: The signals received by each of the SU nodes are affected by Rayleigh fading and thermal noise. We assume that the channel state is constant for the whole slot length. For the analysis, we assume that each SU experiences the same instantaneous PU fading, which is described by a Rayleigh fading process. The SU transmissions are all affected by independent identically distributed Rayleigh fading as well.

\section{B. Physical Layer Model}

We now explain the physical layer model, which consists of PU detection, packet capture, and slot availability, in more detail.

1) PU Detection Errors: Every node of the OSA network at the beginning of every slot will sense to detect the presence of a PU on a given channel. Typically, the detection process is performed based on the observed energy level. This is the most classical detector, which is very easy to implement. However, we are aware of the fact that energy detection might not be the sufficient solution for OSA. (Energy detection performs poorly when the signal power is close to the noise region and there is a noise uncertainty.) For this study, we, however, only need a relation between the detection performance and the scanning overhead. Being well and intuitively understood detection method, for which the equations for detection errors have a closed-form solution, is a well-motivated detection technique for this paper.

Assuming that we are detecting an unknown signal in the presence of white Gaussian noise, the probability of false alarm, given that a PU is not present, is given as [33, eq. (12)]

$$
p_{f}=\frac{\Gamma(\lfloor s W\rfloor, \theta / 2)}{\Gamma(\lfloor s W\rfloor)}
$$

where $W$ is the channel bandwidth; $\theta$ is the detection threshold; and $\Gamma($.$) and \Gamma(.,$.$) are the complete and upper incomplete$ gamma functions, respectively. The probability of detection, given that a $\mathrm{PU}$ is present, is

$$
p_{d}=\frac{1}{\bar{\gamma}} \int_{0}^{\infty} Q_{\lfloor s W\rfloor}(\sqrt{2 \gamma}, \sqrt{\theta}) \exp \left(-\frac{\gamma}{\bar{\gamma}}\right) d \gamma
$$

where Q.(.,.) is the generalized Marcum Q-function, $\bar{\gamma}=P_{P} / N_{0}$ is the mean signal-to-noise ratio (SNR) observed by the $\mathrm{SU}$ energy detector, $\gamma$ is the instantaneous SNR observed by the SU, and $N_{0}$ is the noise level. Equation (2) gives the probability of signal detection in the presence of Gaussian noise only (which is described in this case by the generalized Marcum Q-function) averaged over the fading statistics. (For the Rayleigh fading considered here, the PU received signal power is exponentially distributed.) Equation (2) has a closedform solution given by

$$
\begin{aligned}
& p_{d}=e^{-\frac{\theta}{2}}\left\{\sum_{h=0}^{\lfloor s W\rfloor-2}\right. \frac{\theta^{h}}{h ! 2^{h}}+\left(\frac{1+\bar{\gamma}}{\bar{\gamma}}\right)^{\lfloor s W\rfloor-1} \\
&\left.\times\left[e^{\frac{\theta \bar{\gamma}}{2+2 \bar{\gamma}}}-\sum_{h=0}^{\lfloor s W\rfloor-2} \frac{(\theta \bar{\gamma})^{h}}{h !(2+2 \bar{\gamma})^{h}}\right]\right\}
\end{aligned}
$$

which corrects the typographical error of [33, eq. (16)]. All the parameters used for the derivation of detection errors are also listed in Table II.

2) Packet Capture: Fading causes additional packet loss for the OSA. Since these losses are not specific to the analysis of multichannel MAC designs in the context of OSA, we neglect them. However, fading can lead to a throughput increase for the OSA network in the following scenario. Even if the OSA node has mistakenly detected the channel as free from PU activity, if the received PU signal has been in deep fade, the OSA node might be able to successfully transmit its packet (this effect has been observed first in [34]) due to packet capture. Therefore, we have introduced the packet capture model to account for these phenomena in our analysis.

We assume that $n$ colliding OSA packets with the average received power $P_{S}$ (including the PU interference of the average received power $P_{P}$ ) are coherently added at the OSA node. ${ }^{3}$ In addition, we omit the impact of noise in the capture since interference from competing packets will be the dominant factor in the capture process [35, eq. (20.b)]. Having all the assumptions, we can compute the capture probability for the OSA network, as given in the following proposition:

Proposition 1: Given the coherent addition of signals, with capture threshold $\varphi, n \in[0, N-1]$ interferers, PU interference power $P_{P}$, and SU packet power $P_{S}$, the capture probability is

$$
S_{x, n}(\varphi)=1-\frac{P_{x}(n) \varphi}{P_{S}+P_{x}(n) \varphi}
$$

\footnotetext{
${ }^{3}$ Coherent addition requires phase knowledge of the signals, which might be difficult for the OSA case. However, from the analytical perspective, the noncoherent generalization requires the pdf of the sum of unequally distributed exponential variables, i.e., $n$ variables with average parameter $P_{S}$ and one variable with average parameter $P_{P}$, which has no compact form. Furthermore, the resulting pdf needs to be convoluted with the fading distribution of the test packet and integrated, which introduces another challenge.
} 
where

$$
P_{x}(n)= \begin{cases}P_{P}+n P_{S}, & x=1(\mathrm{PU}+\mathrm{SU} \text { interference }) \\ n P_{S}, & x=2(\mathrm{SU} \text { interference })\end{cases}
$$

Proof: According to [35, eq. (20)], the probability that a transmitted fragment is lost is defined as

$$
F_{Z_{n}}(\varphi)=\int_{0}^{\varphi} d z \int_{0}^{\infty} f_{P_{s}}(z w) f_{P_{n}}(w) d w
$$

where $f_{P_{n}}($.$) is the probability density function (pdf) of the$ interfering signals in a slot, and $f_{P_{s}}($.$) is the pdf of the received$ test packet. Starting from this definition, since we have assumed coherent addition of interfering signals, the sum of the interference power is exponentially distributed with average parameter $P_{x}(n)$ defined as in (5). Then, we have

$$
\begin{aligned}
F_{Z_{n}}(\varphi) & =\int_{0}^{\varphi} d z \int_{0}^{\infty} \frac{w}{P_{S} P_{x}(n)} \exp \left(-w \frac{z P_{x}+P_{S}}{P_{S} P_{x}(n)}\right) d w \\
& =\int_{0}^{\varphi} \frac{P_{S} P_{x}(n)}{\left(z P_{x}(n)+P_{S}\right)^{2}} d z=\frac{P_{x}(n) \varphi}{P_{S}+P_{x}(n) \varphi} .
\end{aligned}
$$

Since $S_{x, n}(\varphi)=1-F_{Z_{n}}(\varphi)$, the proof is complete.

3) Slot Availability: Having the analytical formulas for PU detection errors and packet capture, we can compute the probability that a given slot will be available for SU communication, incorporating all the effects.

Proposition 2: The possible slot availability for the SU node, given $n$ other SU nodes transmitting, is

$\psi(n)=\left(1-q_{p}\right)\left(1-p_{f}\right) S_{2, n}(\varphi)+q_{p}\left(1-p_{d}\right) S_{1, n}(\varphi)$.

Proof: A slot can be available to the SU in two distinct cases: 1) when there is no PU on the channel and 2) when there is one. In both cases, interference effects might reduce the probability that the slot will not be available but only when the detector properly decides on the channel state. Combining these effects altogether, we obtain (8).

\section{Markov Analysis}

In Section III-A, we listed system assumptions that allow one to model the multichannel OSA MAC performance as a Markov model, where the state space gives the number of channels in use for data communication. More specifically, assumptions 2 and 4 define the time granularity of a discrete-time Markov model, and assumptions 3 and 4 relate to the transition probabilities, i.e., when a successful arrangement results in a new data transmission or when an ongoing data transmission is finished. On top of that, assumptions 1 and 5 allow one to embed the impact of PU activity on the MAC performance. In this section, we will describe the Markov model and show how to embed the physical model derived earlier into it. For the analysis, we will focus on one representative OSA MAC from the two OSA MAC groups defined in Section II-B, i.e., DCC for the CC OSA MACs and HCC for the hopping OSA MACs.

1) State Space and Steady-State Channel Occupancy: In the considered discrete Markov chain state, $X_{t}=k$ represents the $2 k$ devices ( $k$ pairs) involved in the transmission at time $t$. The state space is then

$$
\mathcal{S}=\left\{0,1, \ldots, \min \left(\left\lfloor\frac{N}{2}\right\rfloor, M_{D}\right)\right\}
$$

Following this definition of the state space, we can define the steady-state vector of the Markov chain $\Pi=$ $\left\{\pi_{0}, \pi_{1}, \ldots, \pi_{\min \left(|N / 2|, M_{D}\right)}\right\}$. From this, the average utilization per channel, which is considered as the proportion of time when the channel is occupied by the $\mathrm{SU}$, is

$$
\rho=\psi(0) \frac{\sum_{i \in \mathcal{S}} i \pi_{i}}{M_{D}}
$$

where $\psi(0)$ is the slot availability, when a single $\mathrm{SU}$ is accessing the channel for data communication. We note that SU collisions only happen during the control message exchange. In addition, we note that the computed utilization for the nonOSA multichannel case represented by [10, eq. (4)] has to be decreased by the slot availability since we assume that, when the channel becomes busy during the data phase, the SU just waits until the channel becomes available again on that channel. Hence, although the SU is residing on channel $m$ in the steady-state computation, it is not effectively using it. Without that adjustment, the Markov model would consider the PU occupancy as an increase in channel utilization by the SU network. Using (10), we can finally compute the throughput, by which we mean the total throughput of the OSA network as a whole, i.e., the average throughput of one secondary network consisting of multiple SUs, as

$$
\begin{aligned}
R & =M_{C} C \rho \frac{l}{s+l} \\
M_{C} & = \begin{cases}M_{D}-1, & \text { for DCC } \\
M_{D}, & \text { otherwise. }\end{cases}
\end{aligned}
$$

The separate definition of the throughput for DCC comes from the fact that, for this MAC class, one channel is dedicated only to RTS/CTS (control) exchange. Next, in comparison to [10, eq. (9)], the throughput formula has to be reduced by a scanning factor $l /(s+l)$, given that only part of the whole slot is used for effective data communication.

2) Transition Probabilities: To compute the steady-state vector, we need to define the transition probabilities, i.e., the probabilities of starting a new data transmission after a successful connection arrangement or finishing a data transmission when the data packet was sent.

Let $S_{j}^{(k)}$ denote the probability of $j$ new arrangements, given that $k$ pairs are communicating, and $T_{j}^{(k)}$ denote the termination probability of $j$ connections, given that $k$ pairs 
are communicating. Then, the entries of transition probability matrix $\mathbf{P}$ are

$$
p_{k z}= \begin{cases}0, & \text { if } z>k+1 \\ T_{k}^{(0)} S_{k}^{(1)}, & \text { if } z=k+1 \\ T_{k}^{(k-z)} S_{k}^{(0)} & \\ +T_{k}^{(k-z+1)} S_{k}^{(1)}, & \text { if } 0<z \leq k \\ & \text { and } k+z \neq 2 \max \{\mathcal{S}\} \\ T_{k}^{(k-z)} S_{k}^{(0)} & \\ +T_{k}^{(k-z+1)} S_{k}^{(1)} & \\ +T_{k}^{(k-z)} S_{k}^{(1)}, & \text { if } k=z=\max \{\mathcal{S}\} \\ T_{k}^{(k)} S_{k}^{(0)}, & \text { if } z=0 .\end{cases}
$$

We emphasize that (13) corrects the error in transition probabilities of [10, eq. (6)]. In [10], Mo et al. did not consider the particular transmission probability for condition $k=z=$ $\max \{\mathcal{S}\}$, which means that data transfer is not possible even after a successful connection arrangement, because all channels are busy. This happens with probability $T_{k}^{(0)} S_{k}^{(1)}$. When this case is not correctly considered, the resulting matrix $\mathbf{P}$ in [10] is not stochastic, i.e., each row does not sum to 1 , which is a necessary condition for finite-state Markov chains. We will elaborate on this issue more in Section III-D.

Because of the possible PU operation on each of the $M_{D}$ channels, fading effects, and PU detection errors, the definitions of $S_{j}^{(k)}$ and $T_{j}^{(k)}$ given in [10] need to be changed. The respective definitions follow.

3) Termination Probability $T_{k}^{(j)}$ : The probability $T_{k}^{(j)}$ that $j$ connections finish the transfer in a given slot is

$$
T_{k}^{(j)}=\left(\begin{array}{c}
k \\
j
\end{array}\right)\{q \psi(0)\}^{j}\{1-q \psi(0)\}^{k-j}
$$

which means that the nodes involved in a transmission can successfully terminate only when the slot is available for the SUs. We note that we neglect the details of the retransmission policy, which could indeed be included in the termination expression. Our expression is for the simple case where we have an ACK per fragment (one slot) and we just retransmit the fragment in the next available slot if necessary.

4) Arrangement Probability $S_{k}^{(j)}$ : Different OSA MAC classes have different mechanisms in arranging a new connection, which is translated into different probabilities to start a transmission in the Markov model. Here, we will individually derive these important probabilities for each multichannel MAC class.

For DCC and HCC, arrangement probability $S_{k}^{(j)}$ is

$$
S_{k}^{(j)}= \begin{cases}S_{A, x}, & \text { if } j=1 \\ 1-S_{A, x}, & \text { if } j=0 \\ 0, & \text { otherwise }\end{cases}
$$

where $S_{A, x}$ is separately defined for DCC $(x=1)$ and HCC $(x=2)$ in the succeeding sections. a) DCC: In the case of dedicated CC MAC, the average probability of successfully receiving the packet in the presence of interferers is [36]

$$
S_{A, x=1}=\sum_{i=1}^{N-2 k}\left(\begin{array}{c}
N-2 k \\
i
\end{array}\right) p^{i}(1-p)^{N-2 k-i} \psi(i-1) .
$$

Since the successful transmission might happen to only one user, given that $i$ users are competing for the channel at the same slot, summing over all possible amounts of interferers $i$, we obtain the expected probability of successful arrangement. The number of interferers is bounded by $N-2 k$ since $2 k$ users are involved in the data transmission and will not compete for the new arrangement.

Equation (16), however, does not prohibit having more than one sender-receiver SU pair to access the data channel, i.e., more than one user can be successful in capturing the packet. However, since we are particularly interested in the capture effects between PU and SU and, in an OSA setup, the SUs are usually nearby and the PU is far away, we have

$$
S_{A, x=1}=(N-2 k) p(1-p)^{N-2 k-1} \psi(0) .
$$

We note that using (16) in a model will give an upper bound for the OSA network throughput, whereas using (17) will result in a throughput lower bound. For the numerical results, we will proceed with the arrangement probability definition of (17).

b) HCC: In this case, we modify (17), as in [10, Sec. III-B], to take into account the probabilities that the receiver is available and that the channel is not used for data communication. Therefore, we have

$$
S_{A, x=2}=S_{A, x=1} \frac{N-2 k-1}{N-1} \frac{M_{C}-k}{M_{C}} .
$$

To make a fair comparison of HCC OSA MAC with DCC OSA MAC, one needs to take into consideration the switching time. As in [10], this is modeled by modifying the average SU data packet length in the slots as $q^{-1}=q^{-1}((l+s) /(l+s+$ $\left.\left.t_{p}\right)\right)$, where $t_{p}$ is the channel switching time.

\section{Verification of the Analytical Model}

To verify our proposed analytical model, we have developed an event-driven simulator in Matlab and Octave, which closely mimics the conditions assumed in Section III-A. Our simulator allowed the tracking of the channels that have been chosen by the SUs and PUs during their respective connections (which was not possible in the analytical model). Results of the simulations were generated using the method of batch means for $95 \%$ confidence intervals. Each simulation was divided into at least five batches, where each batch contained at least 5000 networkrelated events (see, e.g., [37] for a similar approach of analytical model verification).

1) Comparison of the Proposed Model With [10]: As we have noted in Section III-C2, the transition probability matrix of [10, eq. (6)] for comparing non-OSA multichannel MACs 
was incorrect. Using transition matrix [10, eq. (6)] for $k=$ $\max \{\mathcal{S}\}$, we have

$$
\begin{aligned}
\sum_{i=0}^{k} \mathbf{P}_{k, i}= & T_{k}^{(k)} S_{k}^{(0)}+T_{k}^{(k-1)} S_{k}^{(0)}+T_{k}^{(k)} S_{k}^{(1)}+\cdots \\
& +T_{k}^{(0)} S_{k}^{(0)}+T_{k}^{(1)} S_{k}^{(1)} \\
= & \sum_{j=1}^{k} T_{k}^{(j)}+T_{k}^{(0)} S_{k}^{(0)} \\
= & 1-T_{k}^{(0)}\left(1-S_{k}^{(0)}\right)<1 \text { if } S_{k}^{(0)}<1 .
\end{aligned}
$$

In the model proposed in this paper, the last term of $\sum_{i=0}^{k} \mathbf{P}_{k, i}$ is replaced by $T_{k}^{(0)} S_{k}^{(0)}+T_{k}^{(1)} S_{k}^{(1)}+T_{k}^{(0)} S_{k}^{(1)}=$ $T_{k}^{(0)}+T_{k}^{(1)} S_{k}^{(1)}$, which, after performing simple algebra as in (19), results in $\sum_{i=0}^{k} \mathbf{P}_{k, i}=1$. Interestingly, only in the DCC case is $S_{k}^{(0)}<1$ since, in the HCC connection, arrangement is impossible when all channels are busy $(k=\max \{\mathcal{S}\})$. To observe how severe the error in the original model was, we plot the throughput of DCC for different access probability values $p$ and different levels of PU activity using our simulator, and compared it with our model and that of [10] in Fig. 1. We can clearly see that, for the considered parameter range, the mismatch between the simulations and analysis presented here using [10, eq. (6)] is visible when the access probability results in the highest throughput [approximately $p=[0.05,0.25]$ in Fig. 1(a) and $p=[0.025,0.1]$ in Fig. 1(b)]. In contrast, our proposed model gave a perfect match with the simulations. (All points are within $95 \%$ confidence intervals.)

2) OSA MAC Scaling With PU Activity: Next, we check how the multichannel OSA MACs scale with PU activity; the results are presented in Table III. For each scenario, we assume that the channel access is optimized, and we achieve this by choosing the access probability $p$ that maximizes the throughput. For $q_{p}=[0.1,0.4]$, which are the values of interest, we can see that our model closely matches the simulation results since all values are within $95 \%$ confidence intervals. We note, however, that our model gives a less accurate match with the simulations for very high values of PU activity $q_{p} \geq 0.6$ (as shown in Table III for DCC with $M_{D}=3$ and $N=11$ ). In addition, we have observed that the model is sensitive to the selection of transmission probability $p$, i.e., there is less match with the simulations when the network operates in highly saturated conditions.

From Table III, we expect a linear decrease in throughput for the OSA network when the PU activity increases. We will closely evaluate this in Section IV-B1. We also observe that DCC performs slightly better than HCC for the chosen setup, which is consistent with [10, Fig. 4].

3) Impact of Sensing on OSA MACS: Next, we verify the correctness of the operation of the model as a function of scanning performance in Table IV. While keeping most parameters fixed (see Table IV), we vary the detection threshold $\varphi \in\{14.5,13.9,13.5\} \mathrm{dB}$, which results in different $p_{d}=$ $\{0.93,0.94,0.95\}$. As in the preceding section, we assume that the channel access is optimized by choosing the channel access probability $p$ that results in maximal throughput. We

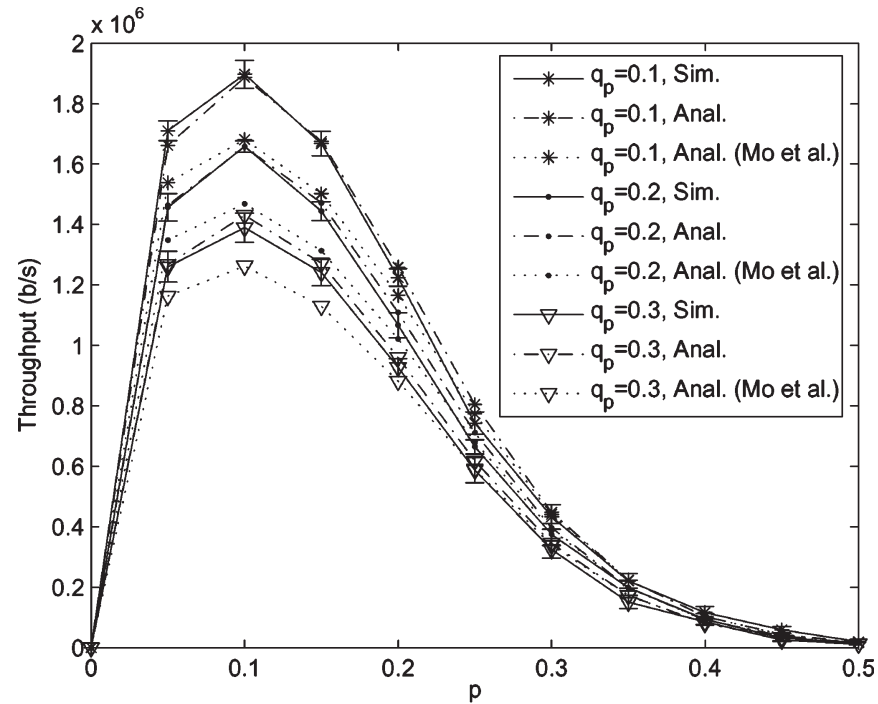

(a)

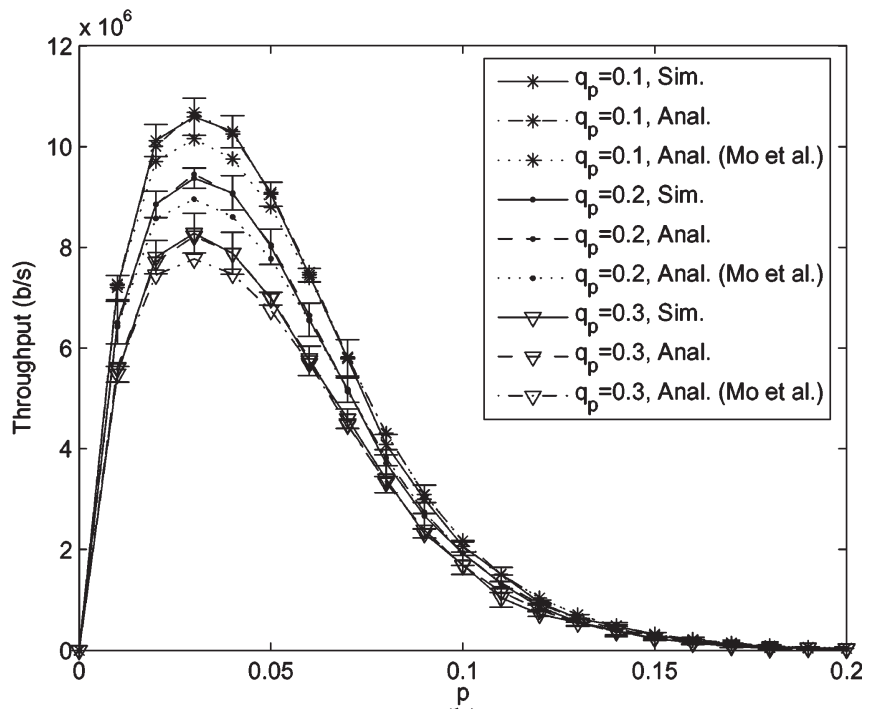

(b)

Fig. 1. Numerical verification of the DCC MAC class throughput with respect to the probability of transmit $p$ and different PU activity values $q_{p}$ for the proposed model and with the use of the transition probabilities of Mo et al. [10]. (a) $M_{D}=3, N=12, q=0.6 \mathrm{kB}$, and $l=812 \mu \mathrm{s}$. (b) $M_{D}=12$, $N=40, q=0.9 \mathrm{kB}$, and $l=200 \mu \mathrm{s}$. Common parameters: $C=2 \mathrm{Mb} / \mathrm{s}, s=$ $20 \mu \mathrm{s}, N_{0}=-90 \mathrm{dBm}, P_{P}=-85 \mathrm{dBm}, P_{S}=-80 \mathrm{dBm}, W=1 \mathrm{MHz}$, $\theta=17.8 \mathrm{~dB}$, and $\varphi=9.5 \mathrm{~dB}$.

perform the verification once for an average PU occupancy of $6.8 \%\left(q_{p}=0.068\right)$, which corresponds to our measured value in [1, Tab. 1], and then for a PU occupancy that is three times larger. Again, our model closely matches the simulation since all results are within the $95 \%$ confidence intervals for all values of the detection performance. As expected, the throughput decreases with an increasing $p_{f}$.

After verifying the important aspects of our model, we proceed in the next section with a more detailed performance analysis using the analytical model only.

\section{E. Numerical Result}

1) Impact of CC Availability: As we have noted in Section II, DCC is the most used OSA MAC. It is therefore 
TABLE III

VERIFICATION OF THE ANALYTICAL MODEL FOR THE SU NETWORK THROUGHPUT $R \mathrm{Mb} / \mathrm{s}$ AS A FUNCTION OF PU ACTIVITY FOR (Top Half) $M_{D}=3, N=12, C=2 \mathrm{Mb} / \mathrm{s}$, AND $l=812 \mu \mathrm{s}$, AND (BotTom Half) $M_{D}=12, N=40, C=6 \mathrm{Mb} / \mathrm{s}$, AND $l=200 \mu \mathrm{s}$. Common Parameters: $s=10 \mu \mathrm{s}, N_{0}=-90 \mathrm{dBm}, P_{P}=-85 \mathrm{dBm}$, $P_{S}=-80 \mathrm{dBm}, W=1 \mathrm{MHz}, \theta=17.8 \mathrm{~dB}$, AND $\varphi=20 \mathrm{~dB}$ (SEE TEXT FOR FURTHER EXPLANATION)

\begin{tabular}{l|l|c||c|c|c}
\hline$q_{p}$ & DCC (sim.) & DCC (anal.) & DCC (sim.) & DCC (anal.) \\
\hline & \multicolumn{2}{|c||}{$q=0.5 \mathrm{kB}$} & \multicolumn{2}{|c}{$q=1 \mathrm{kB}$} \\
\hline 0.10 & $(0.94,1.08)$ & 1.08 & $(1.84,1.94)$ & 1.92 \\
0.25 & $(0.88,0.92)$ & 0.88 & $(1.47,1.65)$ & 1.57 \\
0.40 & $(0.60,0.70)$ & 0.69 & $(1.18,1.25)$ & 1.22 \\
0.60 & $(0.36,0.45)$ & 0.48 & $(0.57,0.70)$ & 0.84 \\
0.80 & $(0.10,0.15)$ & 0.23 & $(0.18,0.30)$ & 0.42 \\
\hline 0.10 & $(4.06,4.40)$ & 4.13 & $(8.24,9.15)$ & 8.46 \\
0.25 & $(3.30,3.52)$ & 3.40 & $(6.50,7.04)$ & 6.90 \\
0.40 & $(2.58,2.75)$ & 2.67 & $(5.23,5.72)$ & 5.46 \\
0.60 & $(1.58,2.07)$ & 1.93 & $(3.27,4.30)$ & 3.96 \\
0.80 & $(0.73,0.97)$ & 0.96 & $(1.15,2.26)$ & 1.97 \\
\hline \hline$q_{p}$ & HCC (sim.) & HCC (anal.) & HCC (sim.) & HCC (anal.) \\
\hline \multicolumn{2}{|c||}{$q=0.5 \mathrm{kB}$} & \multicolumn{2}{c}{$q=1 \mathrm{kB}$} \\
\hline 0.10 & $(0.87,0.96)$ & 0.87 & $(1.37,1.60)$ & 1.40 \\
0.25 & $(0.70,0.79)$ & 0.72 & $(1.13,1.21)$ & 1.15 \\
0.40 & $(0.52,0.61)$ & 0.56 & $(0.81,0.97)$ & 0.91 \\
0.60 & $(0.27,0.34)$ & 0.36 & $(0.44,0.56)$ & 0.58 \\
0.80 & $(0.10,0.14)$ & 0.18 & $(0.16,0.23)$ & 0.30 \\
\hline 0.10 & $(3.86,4.26)$ & 4.10 & $(7.52,8.07)$ & 7.64 \\
0.25 & $(3.38,3.70)$ & 3.38 & $(6.00,6.70)$ & 6.43 \\
0.40 & $(2.65,2.89)$ & 2.65 & $(4.80,5.10)$ & 4.96 \\
0.60 & $(1.56,1.90)$ & 1.76 & $(3.18,3.78)$ & 3.29 \\
0.80 & $(0.85,1.11)$ & 0.88 & $(1.30,1.79)$ & 1.65 \\
\hline
\end{tabular}

TABLE IV

VERIFICATION OF THE ANALYTICAL MODEL FOR THE SU NETWORK Throughrut $R \mathrm{Mb} / \mathrm{s}$ AS A FunCtion of Probability of FALSE ALARM FOR (TOP HALF) $q_{p}=0.068$ AND (BOTTOM HALF) $q_{p}=0.20$. COMMON PARAMETERs: $M_{D}=3, N=12, C=2 \mathrm{Mb} / \mathrm{s}, l=200 \mu \mathrm{s}, s=10 \mu \mathrm{s}$, $t_{p}=100 \mu \mathrm{s}, N_{0}=-90 \mathrm{dBm}, P_{P}=-85 \mathrm{dBm}, P_{S}=-80 \mathrm{dBm}$,

$W=1 \mathrm{MHz}$, AND $\varphi=20 \mathrm{~dB}$ (SEE TEXT FOR FURTHER EXPLANATION)

\begin{tabular}{|c|c|c|c|c|}
\hline$p_{f}$ & DCC (sim.) & DCC (anal.) & DCC (sim.) & DCC (anal.) \\
\hline & \multicolumn{2}{|c|}{$q=0.5 \mathrm{kB}$} & \multicolumn{2}{|c|}{$q=1 \mathrm{kB}$} \\
\hline 0.10 & $(1.11,1.19)$ & 1.13 & $(1.91,2.07)$ & 1.95 \\
\hline 0.20 & $(1.07,1.15)$ & 1.12 & $(1.81,1.89)$ & 1.86 \\
\hline 0.30 & $(1.03,1.10)$ & 1.10 & $(1.63,1.68)$ & 1.73 \\
\hline 0.10 & $(0.88,0.95)$ & 0.96 & $(1.58,1.67)$ & 1.65 \\
\hline 0.20 & $(0.86,0.96)$ & 0.95 & $(1.48,1.58)$ & 1.57 \\
\hline 0.30 & $(0.86,0.93)$ & 0.93 & $(1.39,1.45)$ & 1.46 \\
\hline \multirow[t]{2}{*}{$p_{f}$} & HCC (sim.) & HCC (anal.) & $\mathrm{HCC}(\operatorname{sim})$. & HCC (anal.) \\
\hline & \multicolumn{2}{|c|}{$q=0.5 \mathrm{kB}$} & \multicolumn{2}{|c|}{$q=1 \mathrm{kB}$} \\
\hline 0.10 & $(3.13,3.21)$ & 3.17 & $(3.20,3.24)$ & 3.22 \\
\hline 0.20 & $(2.83,2.88)$ & 2.85 & $(2.84,2.90)$ & 2.90 \\
\hline 0.30 & $(2.43,2.47)$ & 2.46 & $(2.44,2.50)$ & 2.49 \\
\hline 0.10 & $(2.66,2.72)$ & 2.73 & $(2.75,2.79)$ & 2.76 \\
\hline 0.20 & $(2.37,2.45)$ & 2.44 & $(2.44,2.48)$ & 2.48 \\
\hline 0.30 & $(2.05,2.11)$ & 2.11 & $(2.08,2.14)$ & 2.13 \\
\hline
\end{tabular}

crucial to observe in isolation how this particular MAC class behaves. What differentiates DCC from other multichannel MACs is that one channel is solely assigned to control information exchange. In this section, we will show how unavailability of the $\mathrm{CC}$, due to the PU presence on that channel, affects the operation of the DCC.

The result is shown in Fig. 2. We were particularly interested in the relation between data packet length $q^{-1}$ and CC availability $q_{p}$. The physical layer parameters are chosen such that the scanning overhead is minimum (only $1.2 \%$ of the whole slot), simultaneously resulting in high PU detection performance, i.e., $p_{d}>0.91$ and $p_{f}<0.08$. We will show later in Section III-E5

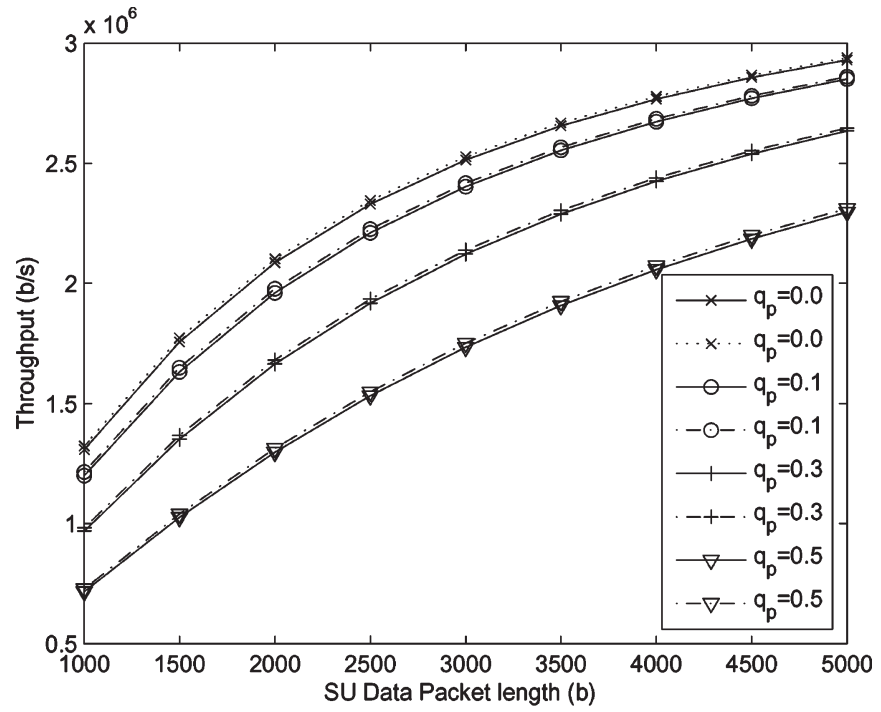

(a)

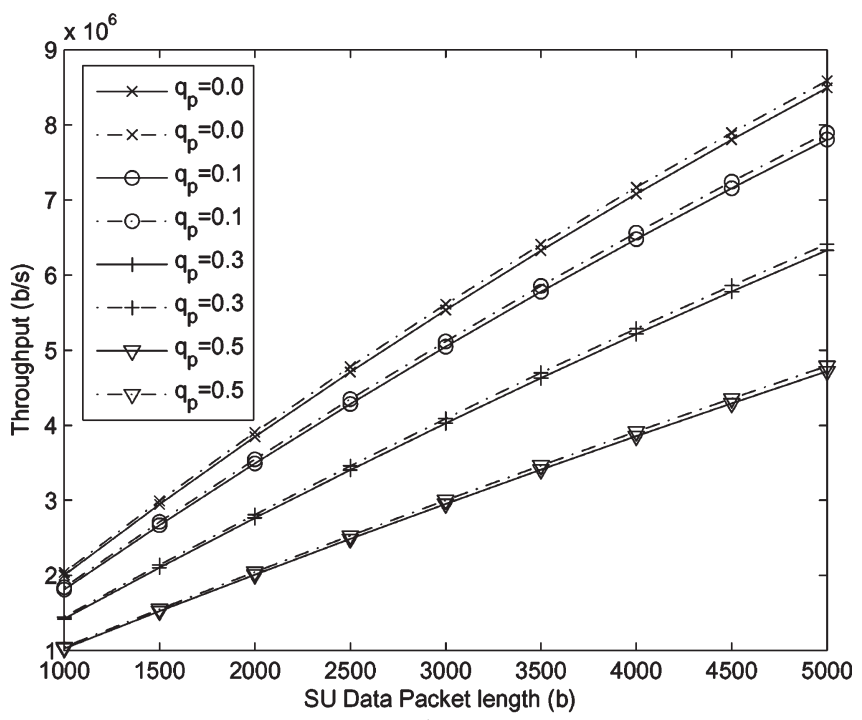

(b)

Fig. 2. Throughput of DCC with respect to $\mathrm{CC}$ availability when data channels are not occupied by PUs using (dashed line) (16) and (solid line) (17). Parameters: $C=2 \mathrm{Mb} / \mathrm{s}, p=e^{-1} / N, l=812 \mu \mathrm{s}, s=10 \mu \mathrm{s}, N_{0}=$ $-90 \mathrm{dBm}, P_{P}=-75 \mathrm{dBm}, P_{S}=-70 \mathrm{dBm}, W=1 \mathrm{MHz}, \theta=14.6 \mathrm{~dB}$, and $\varphi=9.5 \mathrm{~dB}$. (a) $M_{D}=3$ and $N=12$. (b) $M_{D}=12$ and $N=40$.

how to optimize the scanning performance. Moreover, the packet capture probability for SU is less than $20 \%$. We have also assumed that all data channels, except for $\mathrm{CC}$, are unoccupied by the PU, to clearly observe the impact of CC unavailability. Other parameters of the setup used in this section are shown in Fig. 2.

Interestingly, for large packet sizes, even if the $\mathrm{CC}$ is highly unavailable, the network can still achieve moderate or high throughput (see Fig. 2). This is because the SU nodes do not need many new arrangements for long data transmissions, and even if the CC is highly occupied by the PU, it does not significantly affect the operation of the DCC. We clearly see that, when the data packet is small, the impact of CC unavailability is rather high. This means that the SUs can control their impact by controlling the data packet size or connection duration. It is also interesting to note that the impact of CC unavailability on DCC 
is not linear and is more stringent for high unavailability rates, between 0.8 and 1 , since the congestion is worse. In addition, by comparing Fig. 2(a) and (b), we see that the impact is more severe for a higher number of SU channels since, in that case, the congestion on the $\mathrm{CC}$ is worse as well. Again, the SU can adapt to the PU by adapting the number of its used channels. Referring to our measurement results [1, Tab. 1], when the average PU activity was only $6.8 \%$, implementing the $\mathrm{CC}$ on such a channel is possible when the SU network intelligently adapts the packet size and the number of channels in use.

Finally, we have compared the DCC throughputs obtained using (16) and Fig. 2 (dashed line) and (17) and Fig. 2 (solid line). As noted in Section III-C4, the impact of multiple captures on the $\mathrm{CC}$ in our setup, when access probability $p$ is correctly chosen, is very small and would not largely contribute to the collisions on the data channels.

2) OSA MAC Comparison of Throughput Scaling With the Number of Channels: Next, we show how the presence of PUs on all $M_{D}$ channels affects the throughput of each of the two investigated protocols. The result is shown in Fig. 3. As in the previous section, detection quality and capture effects have been set to have a minimum impact on the final result, so that we can clearly see the effects of data channel unavailability on the OSA network throughput. Here, all channels are uniformly and equally occupied by the PU, including the CC for DCC. The number of SUs in the setup is always three times greater than the number of channels. Obviously, the higher the PU activity, the lower the throughput for each of the MAC classes. In Fig. 3(a) and (b), we see that, for a large number of channels, DCC is always better than HCC, independent of the SU data packet size. The reason HCC scales slower is it always wastes channel access time since it can only access a channel every $M_{D}$ slots. For both DCC and HCC, the throughput increase slows down with the increase in the number of channels. HCC saturates because of the slot waste while hopping on other channels, whereas DCC saturates, because the CC gets congested. Comparing Fig. 3 with [10, Fig. 3], we observe the same trends as in the non-OSA case. Our numerical findings (not shown in Fig. 3) show a linear increase in throughput between different PU occupancies. Later, in Section IV-B1, we will investigate the impact of PU activity on the throughput in more detail, analyzing all four OSA MAC classes and taking into account more networking aspects, e.g., queuing.

3) OSA MAC Comparison of Throughput Scaling With the Number of SUs: In the next experiment, we have investigated how an increase in the number of SUs in the OSA network affects the secondary network throughput. We fixed the number of channels and the probability of transmission and varied only the number of users $N$. The results are shown in Fig. 4.

As expected, with the increase in SUs, the network throughput decreases for all the protocols. However, the impact on throughput is more stringent for DCC when the number of users is close to the number of channels [see Fig. 4(a) (left)]. When the number of SUs is approximately twice the number of channels, irrespective of the PU activity, the network throughput for both protocols is more or less the same.

4) PU Detection Quality and Packet Capture: As we have noted earlier, the purpose of introducing capture effects into the

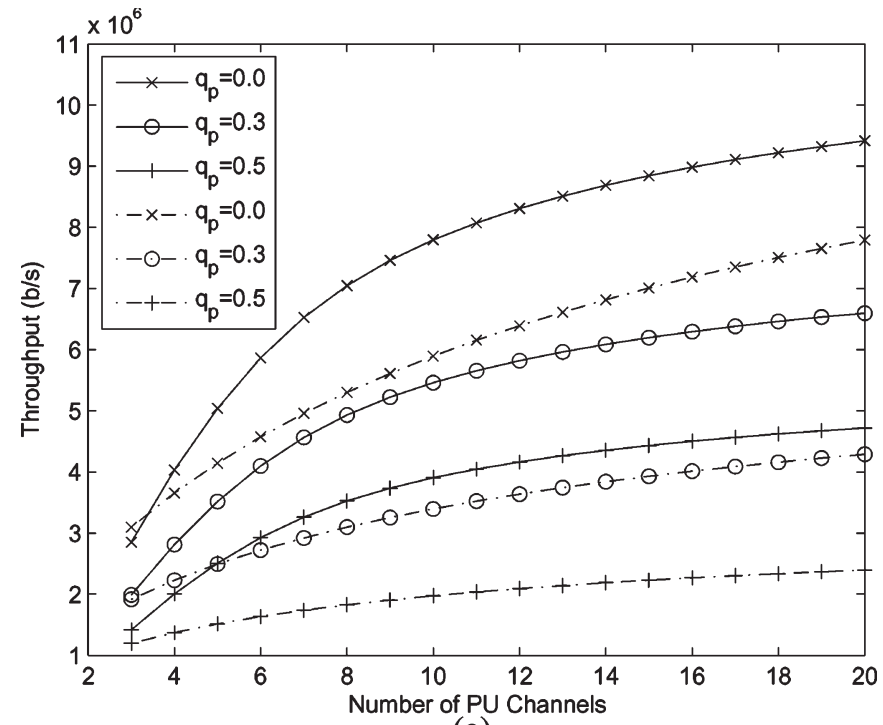

(a)

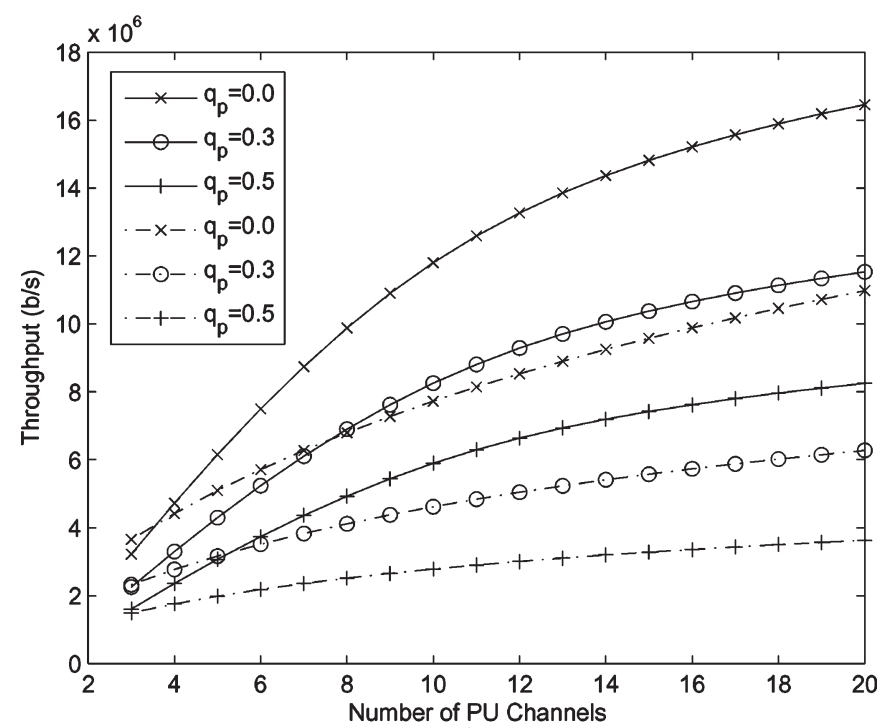

(b)

Fig. 3. Throughputs of (solid line) DCC and (dotted line) HCC as a function of the number of channels for different PU activities on each channel and (a) 5- and (b) 10-kB SU data average packet sizes. The values of $C, l, s, N_{0}$, $P_{P}, P_{S}, W, \theta, \varphi$, and $p$ are the same as those in Fig. 2(a), and $t_{p}=100 \mu \mathrm{s}$. The number of users is always three times larger than the number of channels.

model is to investigate the impact of the following phenomenon on the system performance. Even when the channel has been detected by the OSA network, as occupied by the PUs, depending on the channel conditions, the OSA network can still successfully transmit the packet. Similarly, capture can relax the collisions between the SU nodes on the CC.

We have performed two numerical experiments, for which the results are given in Fig. 5, with the parameter setup presented therein. In the first experiment [see Fig. 5(a)], we have computed how different capture probabilities affect the throughputs of DCC and HCC. We have set the PU detector performance, such that it resulted in a very low probability of false alarm $p_{f}=10^{-13.6}$ and low probability of detection $p_{d}=0.63$. We have set $p_{d}$ such that the phenomenon previously described will clearly be visible. We varied the capture threshold, resulting in a very high capture probability (leftmost 


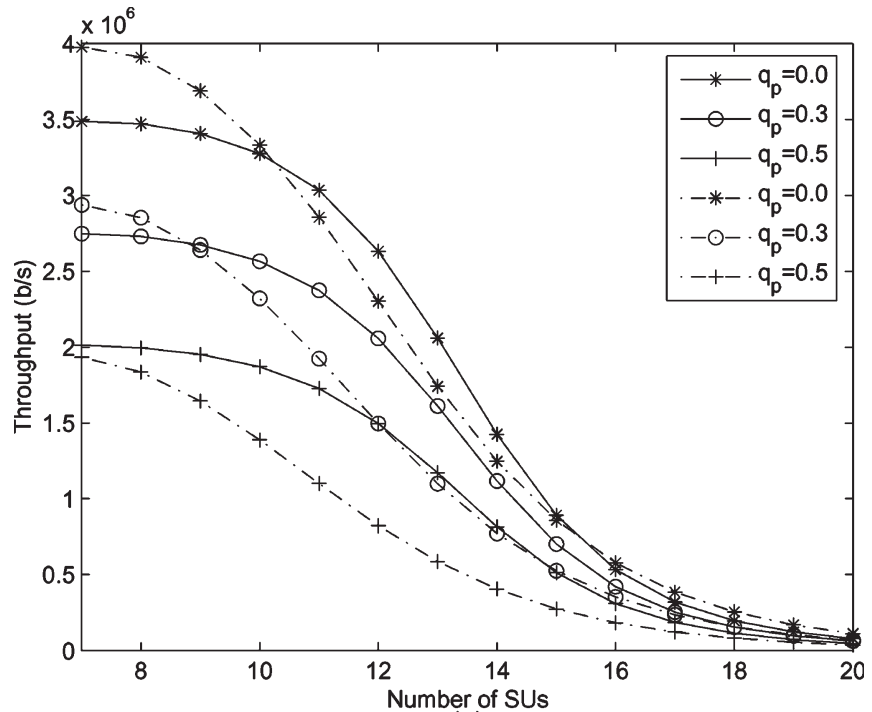

(a)

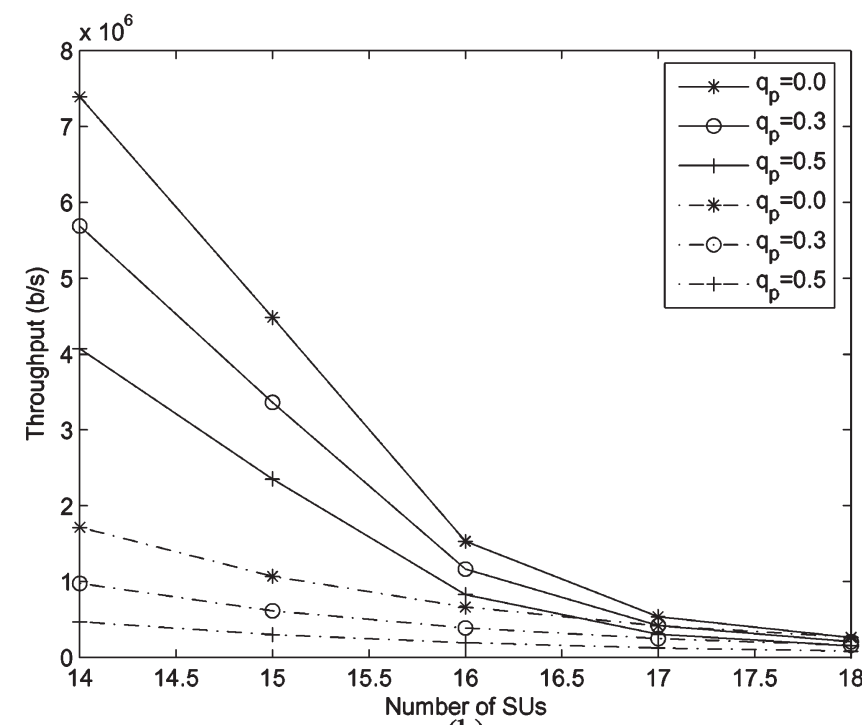

(b)

Fig. 4. Throughputs of (solid line) DCC and (dotted line) HCC as a function of the number of SUs for different PU activities on each channel and (a) $M_{D}=3$ and (b) $M_{D}=12$. Parameters: $C=2 \mathrm{Mb} / \mathrm{s}, p=e^{-1}, l=$ $812 \mu \mathrm{s}, s=1 \mu \mathrm{s}, N_{0}=-90 \mathrm{dBm}, P_{P}=-75 \mathrm{dBm}, P_{S}=-70 \mathrm{dBm}$, $W=1 \mathrm{MHz}, \theta=14.6 \mathrm{~dB}, \varphi=9.5 \mathrm{~dB}, q=1 \mathrm{kB}$, and $t_{p}=100 \mu \mathrm{s}$.

part of the plot) to almost no capture (rightmost part of the plot). The essential observation is that, when the capture probability is very high, it positively affects all protocols. However, when the capture probability drops to a normal level (1\%-5\%), its effect is negligible. Particularly, the positive effect of the capture in the presence of PU interference is more visible when the PU activity is higher, i.e., the slope of the throughput curve is steeper with higher $q_{p}$, for both of the analyzed protocols.

In the second experiment [see Fig. 5(b)], we have observed how a potential capture of the PU might affect the SU network throughput. During this time, we have varied the PU interference power for the same values of $s$ as in Fig. 5(a). Varying the PU power results in varying detection quality, i.e., for the considered case $p_{d}=[0.24,0.99], p_{f}=10^{-13.6}$. The capture is mostly visible when the detection quality is small, i.e., when

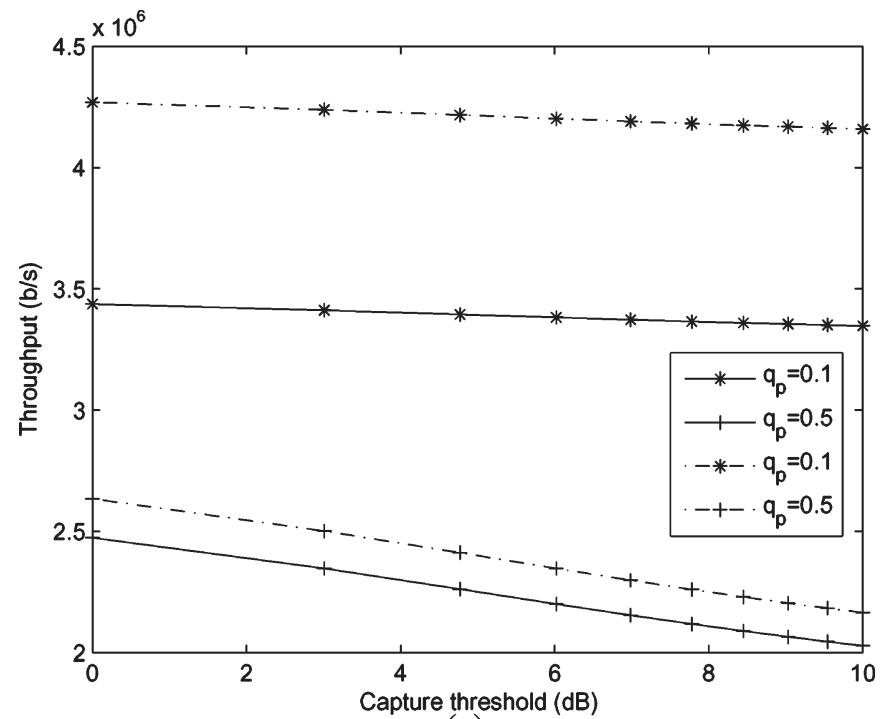

(a)

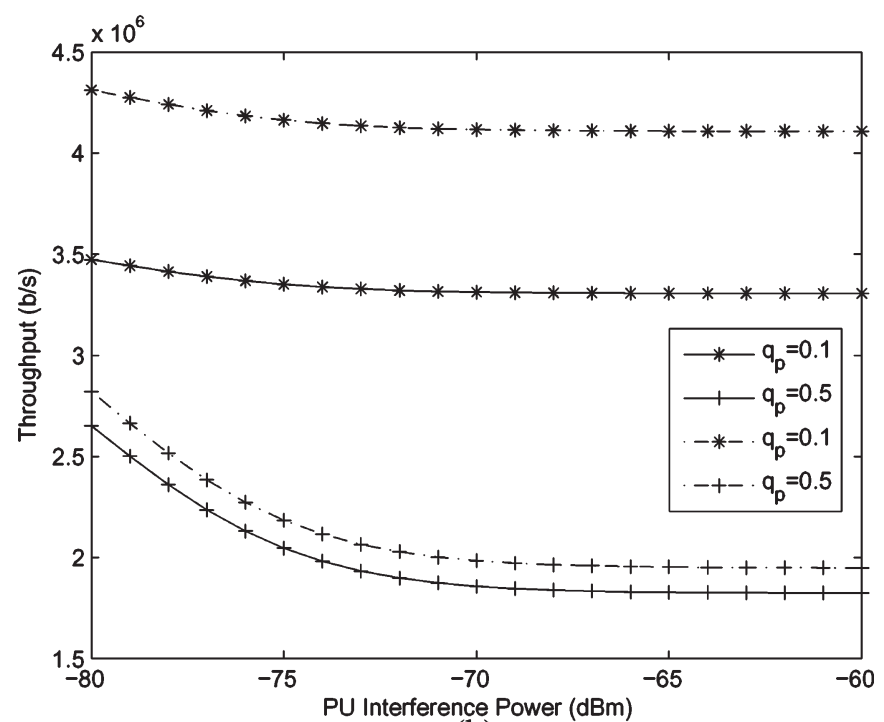

(b)

Fig. 5. Impact of capture effects on the OSA MAC throughput for two different PU activities $q_{p}$. (a) Throughputs of (solid line) DCC and (dotted line) $\mathrm{HCC}$ as a function of capture threshold $\varphi$ for fixed $P_{P}=-75 \mathrm{~dB}$. (b) Throughputs of (solid line) DCC and (dotted line) HCC as a function of PU interference power for fixed capture threshold $\varphi=20 \mathrm{~dB}$. Parameters assumed in both plots: $M_{D}=3, N=20, C=2 \mathrm{Mb} / \mathrm{s}, l=812 \mu \mathrm{s}, s=1 \mu \mathrm{s}$, $N_{0}=-90 \mathrm{dBm}, P_{S}=-70 \mathrm{dBm}, \theta=19 \mathrm{~dB}, W=1 \mathrm{MHz}, t_{p}=100 \mu \mathrm{s}$, $1 / q=10 \mathrm{kB}$, and $p=e^{-1} / N$.

simultaneous transmissions of SU and PU are more probable. With the increase in detection quality, the effect of capture also becomes negligible. With the increase in PU activity, the capture effect becomes less visible. Therefore, the conclusion is that, when the detector is designed sufficiently well, capture from the PU will not increase the SU network throughput as high as that when new PU channels are added to the OSA network [see the results in Section III-E2]. Thus, due to the small effect of the capture on the obtained throughput in the chosen scenarios, we will omit this effect in the simulation model that will be presented later.

5) Impact of Scanning Length: Since the scanning length is a crucial parameter of every OSA system, we quantify how it impacts the performance of multichannel OSA MACs. For 


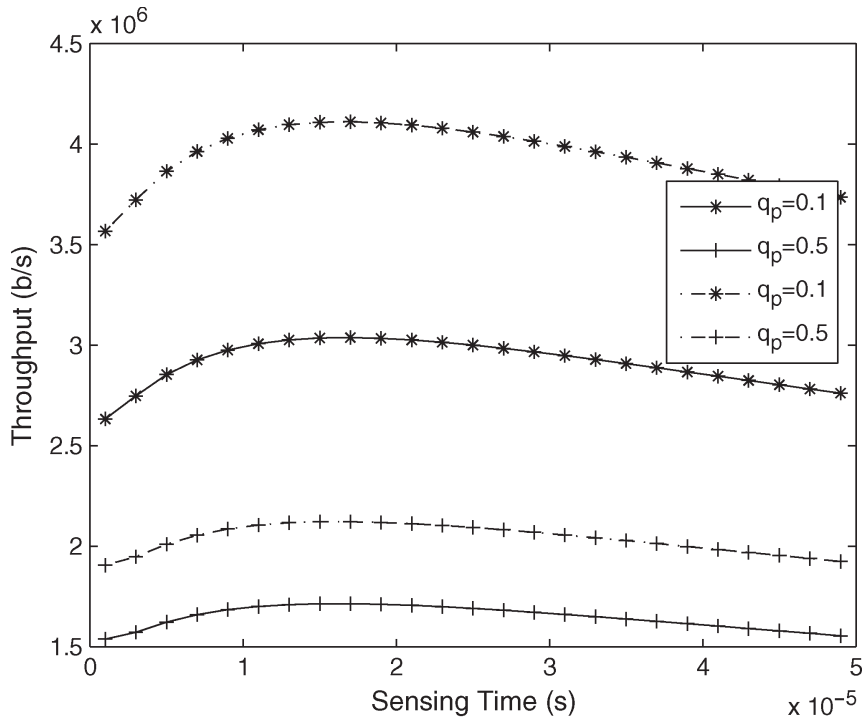

(a)

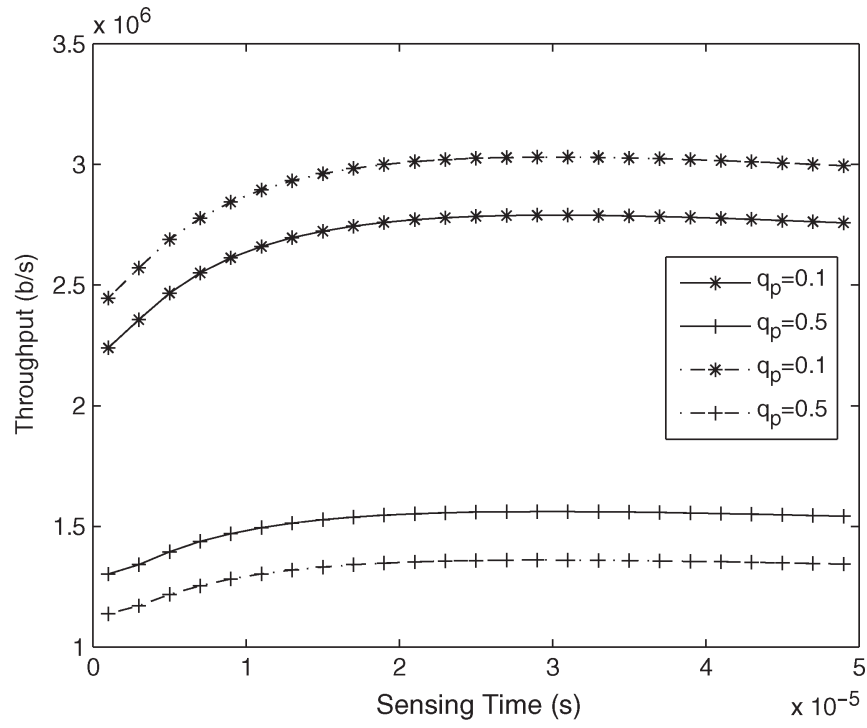

(b)

Fig. 6. Throughputs of (solid line) DCC and (dashed line) HCC as a function of scanning length $s$ for different channel availability rates and (a) $l=$ $200 \mu \mathrm{s}$ and (b) $l=812 \mu$ s slot sizes. $W=1 \mathrm{MHz}, P_{S}=-80 \mathrm{dBm}, P_{P}=$ $-85 \mathrm{dBm}, N_{0}=-90 \mathrm{dBm}, \varphi=9.5 \mathrm{~dB}, N=12, M_{D}=3, C=2 \mathrm{Mb} / \mathrm{s}$, $p=e^{-1} / N, 1 / q=5 \mathrm{kB}$ data packet size, and $t_{p}=100 \mu \mathrm{s}$.

energy detection, increasing the observation time improves the detection performance, i.e., it improves $p_{d}$, simultaneously decreasing $p_{f}$ (see Section III-B1). For the given channel bandwidth $W$ and PU interference power $P_{P}$, we have varied the observation time $s=[1,50] \mu \mathrm{s}$. Simultaneously, we have adapted the sensing threshold as $\theta=10^{7.31} \mathrm{~s} \mu \mathrm{s}^{-1}$ (linear value), which resulted in the following detection performance ranges for the considered range of $s: p_{f}=[0.23,0.02]$ and $p_{d}=[0.82,0.93]$. For these detector operating conditions, we have plotted the throughputs of DCC and HCC in the case of $M_{D}=3, N=12$, and two different slot lengths $l$. The results are shown in Fig. 6.

The most essential observation is that, with the increase in observation time, the throughput first increases due to lower false alarm probabilities but then decreases again. This is due to the fact that scanning length overhead $s$ starts to dominate over the usable slot $l$, reducing the effective throughput of the SU [see (11)]. This is particularly visible for small slot sizes [compare Fig. 6(a) and (b)]. With the highest detection performance for $s=50 \mu$ s in the considered setup, sensing constitutes $9 \%$ of an $l=812 \mu \mathrm{s} \mathrm{slot}$, whereas, for $l=200 \mu \mathrm{s}$, it results in 25\% overhead. Therefore, scanning period $s$ should be carefully chosen for any OSA multichannel MAC since improving the detection performance for decreasing $p_{f}$ does not always improve throughput. Every OSA MAC class is equally affected by this phenomenon. We can therefore formulate the optimization problem as

$$
\max R \text { subject to } p_{d} \geq \alpha
$$

where $\alpha$ is a detection constraint that the OSA network needs to fulfil while accessing PU channels. The parameter that we want to optimize is $R$ with respect to $s$. We know that $p_{d}$ is a nonlinear steplike function, where the computation of this probability is in summation form and $p_{d} \rightarrow 1$ as $h$ in (3) increases. We also know that $R$ is a function of $p_{f}$. Finding an optimal solution in this form is difficult and involves the use of heuristics such as finding, first, a convex envelope and then the optimal value of $p_{d}$ that satisfies $\alpha$ [38], which is beyond the scope of this paper. However, we can think of the other simple heuristic for solving (20). First, we find $h_{\max }=\lfloor s W\rfloor-2$ that fulfills $p_{d}=\alpha$ and compute $R$ using (11). We then increase $h_{\max }$ by 1 and compute the new throughput. If the new throughput is larger than the old throughput, we increase $h_{\max }$; otherwise, we terminate, and the resulting $R$ from the last step is the optimal throughput $R_{\mathrm{opt}}$. The optimization procedure is also depicted in Algorithm 1. We have performed a similar procedure in Fig. 6, where we compute $R$ for every $s$ in the considered range. Then, the maximum value of the throughput obtained in the figure transforms to the optimal sensing time. Please note that, as we have mentioned before, a big overhead for the slot occurs in the upper range of $s$ in Fig. 6; therefore, $s$ should also be upper bounded to reasonable values, e.g., less than $50 \mu \mathrm{s}$, corresponding to the data part of slot $l$.

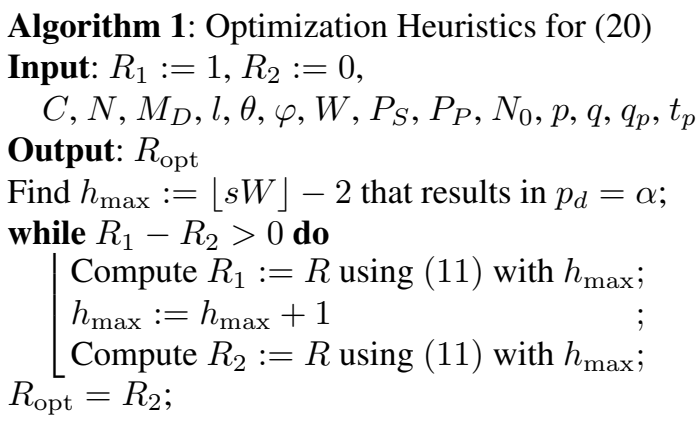

\section{Simulation}

In this section, we discuss the simulation results to compare the four different OSA MAC designs. In the simulation model, we introduce properties such as queuing and a more detailed PU activity model, which were difficult to analytically assess. 
First, we study the throughput for the four OSA MAC designs introduced earlier. Simulation results are given as a function of the sensing performance and PU activity, which is modeled using average channel utilization and the PU packet length. Next, we focus on simulating the delay performance for each of the four MAC classes, again as a function of PU activity and scanning performance. Finally, we measure the expected collision probability with the PU, which we consider to be an important performance metric in the context of OSA networking. We start with introducing the simulation setup.

\section{A. Simulator Description}

We have developed a coarse-grained time-slotted simulator in Matlab, which extends the work of Mo et al. [10] with OSArelated features, such as PU traffic and PU detection. Matlab was the design choice since we needed a rapid-prototyping functionality and overall control over the simulator development process. The simulation environment is coarse grained since it omits all the transient effects related to connection arrangement, i.e., the backoff mechanism. This simplification gives enough information on the interactions between individual SUs and between SUs and PUs while resulting in a fast development time. The smallest time scale observed in the simulator is the RTS/CTS transaction scale, which means that the smallest time slot considered is long enough to transmit an RTS/CTS control handshake. This interaction is fully described by the probability of a new successful connection arrangement $p_{s}$, which captures collision effects due to backoff mechanisms in a simple way. Probability $p_{s}$ is related to the probability $p$ of accessing the channel in the analytical model of Section III. Similar to the assumptions taken in [10], we assume that the probability of having a successful contention in the OSA network is $p_{s}=e^{-1}$, which mimics the optimal operation of the slotted ALOHA protocol. The justification for the introduction of such simulator is presented in more detail in [10, Sec. V].

We now summarize the simulator features, which are grouped in two sets: 1) multichannel-MAC-related aspects and 2) OSA-related features. The multichannel-MAC-related features are given here.

1) Queuing: In the analytical model, no assumption on the buffering has been taken since only the throughput was modeled. The simulator implements packet buffering, and only backlogged packets can contend for the medium. The queuing delay is monitored for the delay analysis.

2) Traffic generation: In the simulator, we have assumed fixed-length packets (five slots or 1024 bytes) for the OSA network, whereas the interarrival times are geometrically distributed.

3) SPCC model: As in [10], the simulator implements contention resolution between multiple SPCC pairs that use the same data channel, when all data channels are utilized.

4) Receiver selection: In the simulation, we assume that each receiver has only one transmitter. Although the case where multiple transmitters contend for a single receiver has been studied in [10], we believe that it is not important to our study, which particularly focuses on the impact of PU traffic on the OSA network.
The OSA-related features of the simulator are given here.

1) Channel occupancy: Each channel is assumed to be occupied with PU packet-based traffic, and the average PU occupancy level is the same for all the considered channels. The distribution of the PU traffic is described by a geometric on/off process. The simulator allows one to vary the average length of the channel on and off periods. The difference, on average, in the period length for the same average PU channel utilization is denoted as $P U$ burstiness, where more burstiness represents shorter average on times. We vary the PU packet size from 10 to 100 slots.

2) Detection errors: As in the analytical model of Section III, every slot is preceded by a scanning period of varying length, after which the OSA node individually decides about the channel occupancy. Detection performance is a function of the length of the scanning period, the noise and PU signal levels, and the fading process. We use the same energy detection model described by (1) and (2). For simplicity, we assume that every OSA node observes the same propagation conditions, such that the PU occupancy decision is the same for all OSA nodes. The parameters used were given as follows: channel bandwidth $W=1 \mathrm{MHz}$, PU interference power $P_{P}=$ $-85 \mathrm{dBm}, N_{0}=-90 \mathrm{dBm}$, and $\theta=10^{7.31} \mathrm{~s} / \mu \mathrm{s}^{-1}$ (linear value). For these settings, we simulated a scanning performance ranging from $p_{f}=[0.2,0.01]$ and $p_{d}=$ $[0.89,0.93]$.

3) Interference to PU: Since the level of harm to the PU introduced by the OSA network is of utmost importance in the context of OSA networks, we monitor the probability of PU interference (collision) in the simulator.

4) Fading: Since the capture effect was shown to be small if $p_{d}$ is sufficiently high, we did not embed capture in the simulation model.

\section{B. Simulation Result}

Having introduced the simulation setup, we now present the simulation results. The focus is on throughput analysis, delay analysis, and interference to the PU.

1) OSA Network Throughput Analysis: To assess the maximum possible throughput that an OSA network can achieve, we simulate the saturation conditions. In Fig. 7, the OSA network throughput is shown to linearly decrease with PU channel utilization, for all four considered MAC designs. This linear decrease with PU activity is expected since each slot occupied by the PU cannot be used by the OSA network. Since not all OSA MAC designs access the channel the same way, they grab the available white spaces with varying efficiencies. This explains the different slopes for the different MAC designs. In Fig. 7(a), we plot the throughput for different scanning lengths, resulting in different performances of the PU detection. As mentioned earlier in Section III, a careful optimization of the scanning performance can result in a significant throughput increase. This is also visible in Fig. 7(a), and the throughput penalty for worse scanning performance is similar for the four MACs. 


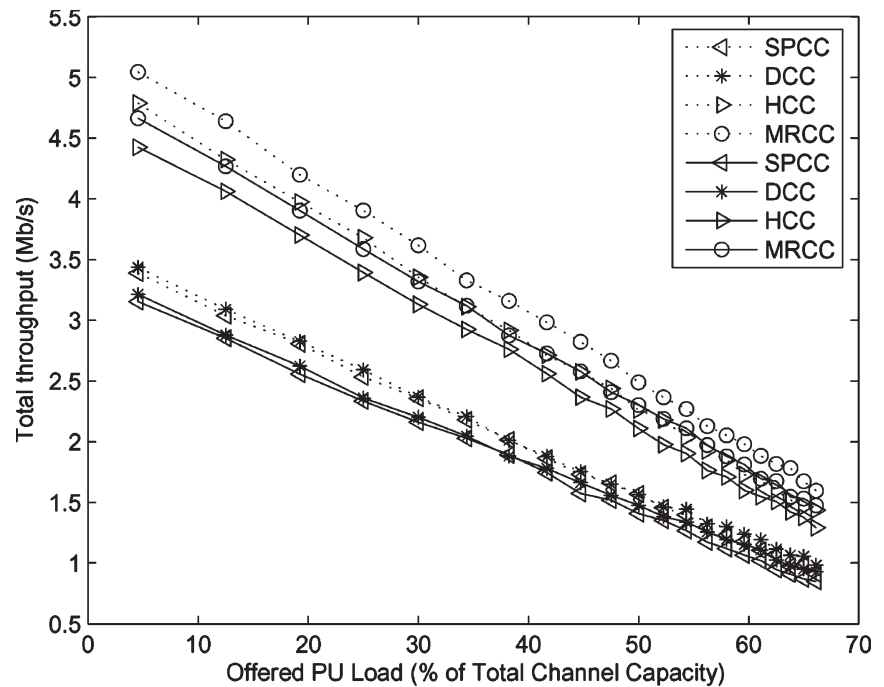

(a)

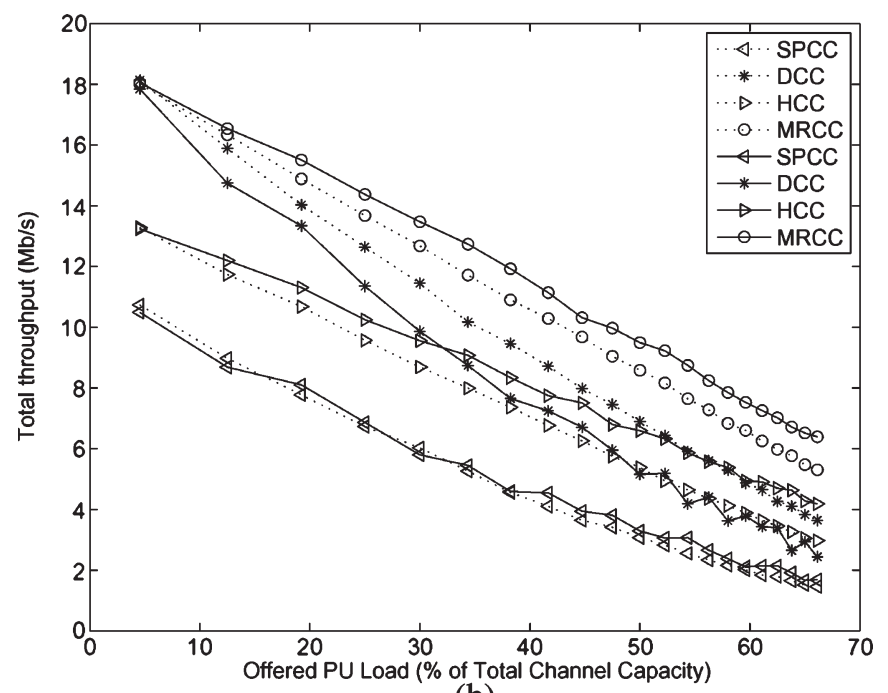

(b)

Fig. 7. Simulated impact of the PU channel occupancy on the throughput of the OSA network exploiting different OSA MACs. (a) $M_{C}=3$ channels and $N=20$ OSA users with different PU detections (solid line: $p_{f}=0.2$ and $p_{d}=0.89$; dashed line: $p_{f}=0.01$ and $p_{d}=0.93$ ) and a PU average packet size of ten slots. (b) $M_{D}=12$ channels and $N=40$ OSA users with different PU packet sizes (dashed line: 10 slots; solid line: 100 slots) and good PU sensing with $p_{f}=0.01$ and $p_{d}=0.93$. Common parameters: $C=2 \mathrm{Mb} / \mathrm{s}$ and $l=812 \mu \mathrm{s}$.

We plot the impact of varying the PU packet size in Fig. 7(b), for a scenario with $M_{D}=12$ channels and $N=40$ OSA users. Surprisingly, HCC and MRCC achieved a larger throughput when the PU packet size is larger (solid line). This is because those MAC protocols contend on the same channel that they will use for the following data transmission if the contention was successful. If the channel is not free, they just hop to another channel, and if they find that the next channel is free, they will be able to send their data packets with high probability if the PU on and off times are longer. DCC, however, contends on another channel and needs to be lucky to find both the control and data channels free from PU activity. A successful contention for DCC does not mean that the chosen data channel is free. (We do not monitor the data-channel PU activity in the simple MAC designs considered.) As a result,

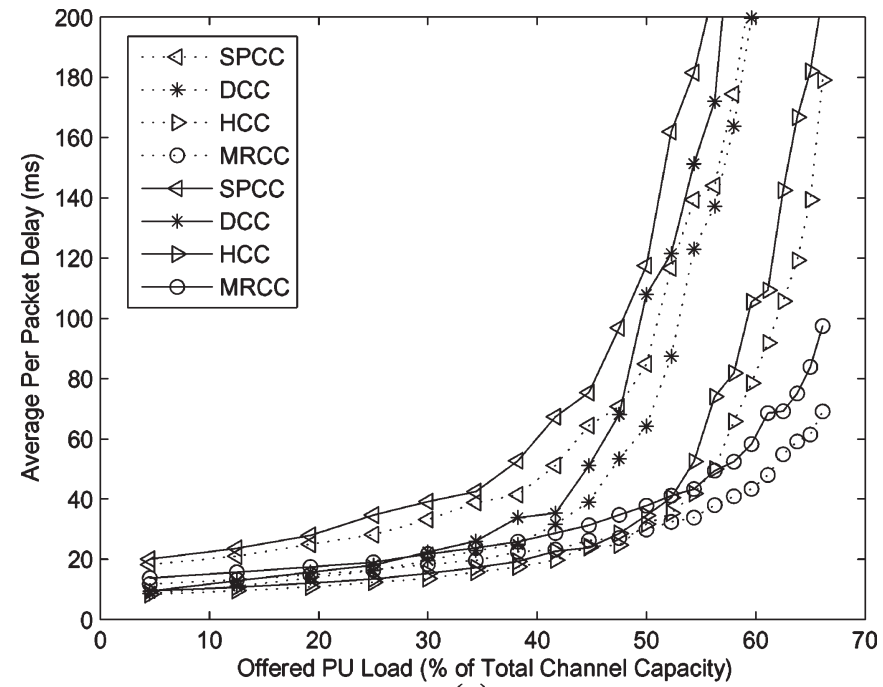

(a)

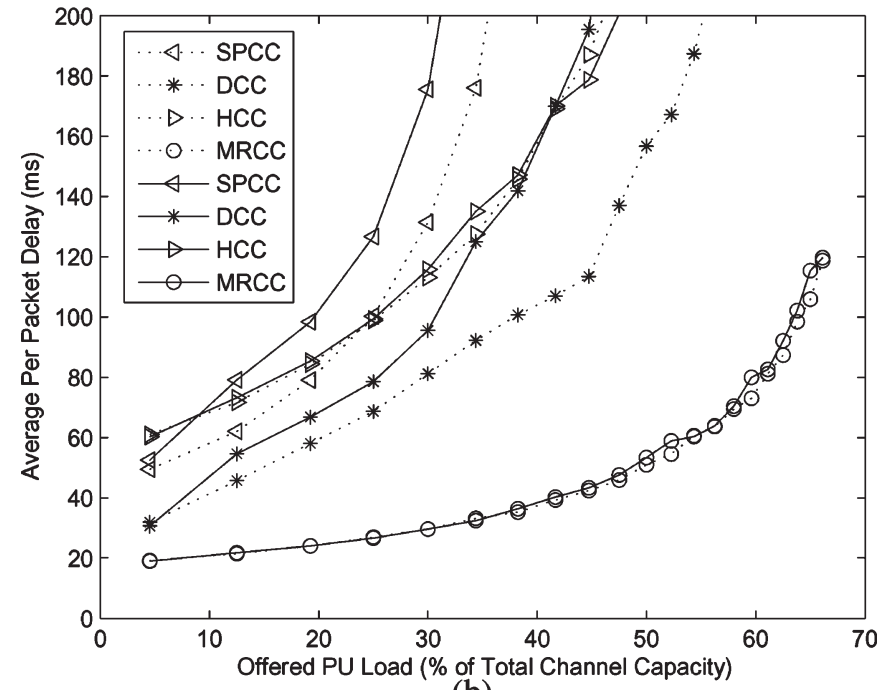

(b)

Fig. 8. Simulated impact of PU channel occupancy on the OSA network delay for different OSA MACs. (a) $M_{D}=3$ channels and $N=20$ OSA users with different PU detections (solid line: $p_{f}=0.2$ and $p_{d}=0.89$; dashed line: $p_{f}=0.01$ and $p_{d}=0.93$ ) and a PU average packet size of ten slots. (b) $M_{D}=12$ channels and $N=40$ OSA users with different PU packet sizes (dashed line: 10 slots; solid line: 100 slots) and good PU sensing with $p_{f}=0.01$ and $p_{d}=0.93$. Common parameters: $C=2 \mathrm{Mb} / \mathrm{s}, l=812 \mu \mathrm{s}$.

DCC's performance suffers much when the PU packet sizes are longer. We also note that, in the simulations, we put the stick limit [10] to 50 slots, which means that, if the data packet was not sent after 50 slots, a new connection arrangement is needed.

2) OSA Network Delay Analysis: To observe the impact of PU occupancy on the OSA network delay, we monitor the OSA network delay for a scenario where the load of the OSA network is $15 \%$ of the total channel capacity in Fig. 8. First, we study a scenario of $M_{D}=3$ channels, with $N=20$ OSA users in Fig. 8(a). In this case, a total load of $15 \%$ of the total channel capacity is equivalent to $45 \mathrm{~kb} / \mathrm{s}$ per flow. When the PU occupancy increases, all protocols suffer from an increased network delay. The impact is, however, the largest for SPCC and DCC since they employ a dedicated channel for control that is prone to congestion. In Fig. 8(a), we also see the impact of the scanning 
performance on the delay. Similar to the throughput analysis, we see that a suboptimal scanning significantly increases the delay for all MAC designs.

In Fig. 8(b), we study the delay for a network with $M_{D}=$ 12 channels and $N=40$ users. In this scenario, $15 \%$ of the total channel resource is also used, which corresponds to $90 \mathrm{~kb} / \mathrm{s}$ per flow. We note that the delay performance of MRCC is similar to that in the first scenario, because the load is also $15 \%$. SPCC and DCC, however, suffer from a larger delay since their $\mathrm{CC}$ becomes more congested when the number of data channels increases. HCC, on the other hand, suboptimally performs since it visits every channel only every 12 slots, and since the packet size is only five slots, every channel is not occupied during the remaining $12-5-1=6$ slots.

In Fig. 8(b), we also study the impact of the PU on and off time distribution. Interestingly, the delay is larger when the PU occupancy increases, because, when the PU occupies the channel for a longer time, the OSA nodes have to wait longer until they can access the channel. Another observation is that the MRCC and HCC MACs are not so sensitive to the packet size of the PU network, because they perform contention on the same channel as the data transmission. When the channel is found to be idle, they can both contend and immediately send data packets if contention was successful. Because of the burstiness, when idle, the PU channel remains idle for the remaining five slots with a high probability. As a result, both protocols hardly have to wait for the long PU packets to be transmitted first since they simply hop to the next channel when a channel is occupied. Since the 12 channels are assumed to be independent, one of the next hops will end up in an idle channel. Again, SPCC performs worst among all the considered classes.

3) OSA Network Impact on PU: The last simulation study focuses on the probability of harming the PU, which is a new and very important performance metric in the OSA context. We define harm to the PU as the probability that a slot occupied by a PU transmission is also used by the SU, causing part of the PU data packet to be lost. For that, we consider a network of $M_{D}=3$ channels with $N=20$ OSA users. The PU occupies the channel $50 \%$ of the time, and the on times are 10 or 100 slots. We simulate a scenario where the total OSA network load is $15 \%$ of the total channel capacity. All differences in interference are hence due to the way the channel access is organized. The results are given in Fig. 9(a). The curves are obtained by varying the probability of false alarm $p_{f}$ from 0.01 to 0.2 , and the considered energy detection model is used to adjust $p_{d}$ and the scanning overhead. With increasing $p_{f}$, detection performance $p_{d}$ decreases, causing more interference to the PU, as expected. $p_{d}$ ranges from 0.93 to 0.89 . As shown in Fig. 9(a), the true impact on the PU is always low (the best $p_{d}$ would result in 7\% harm) since the OSA network does not use all the available channel resources. In addition, interestingly, we see that the interference to the PU significantly decreases when the PU activity in a given slot depends on the activity of that PU in the previous slot. If such dependence is the case, most of the SU packets will not be sent, because the RTS/CTS exchange before the data packet could not be sent as well, preventing more potential harm to the PU during data

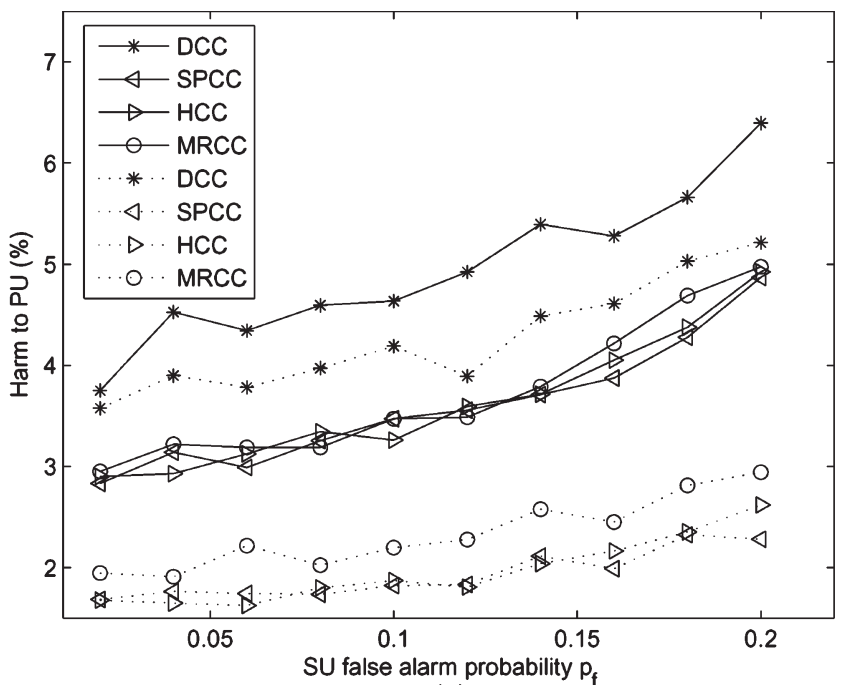

(a)

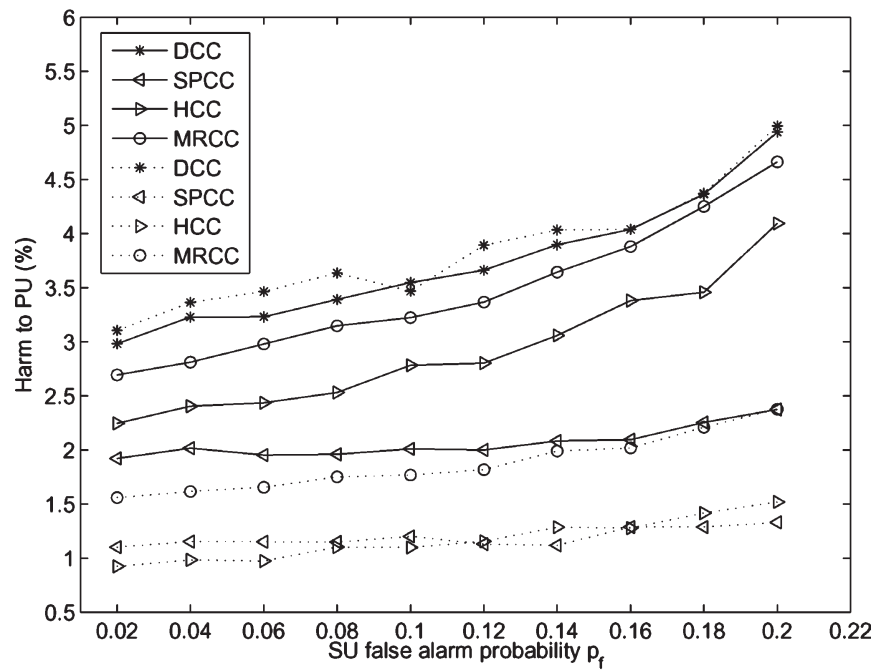

(b)

Fig. 9. Interference to the PU for the four OSA MAC protocols as a function of $p_{f}$ computed from the analytical model. The PU on and off time considered are (solid line) ten slots and (dashed line) 100 slots, for a PU occupancy of 50\%. (a) $M_{D}=3$ channels and $N=20$ users with $15 \%$ channel load. (b) $M_{D}=$ 12 channels and $N=40$ users with $15 \%$ channel load. Common parameters: $C=2 \mathrm{Mb} / \mathrm{s}$ and $l=812 \mu \mathrm{s}$.

activity. This is more visible for MRCC and HCC since those protocols perform network arrangement and data transmission on the same channel. As a result, the probability harming the PU during a data packet is much lower since this can only happen if the PU was not detected during the control phase. When the PU on and off times are large, the probability that PU activity changes during the SU data transfer is small. We also note that DCC does not benefit as much from this, because DCC transmits data and control messages on different channels.

We also plot the PU harm for a scenario with a large number of channels and users in Fig. 9(b). The same load of $15 \%$ is used, and as such, the probability of harming the PU does not increase, although more channels are in use. Compared to the previous scenario, we see that SPCC performs better, but this is due to the fact that SPCC achieves a throughput that is lower than $15 \%$ of the total capacity. (We recall that, 
during the control phase, $M_{D}-1$ of the channels cannot be used.) As such, the channel is less frequently accessed, and the probability of harming the PU is low. Next, we see that MRCC interferes with the PU more than HCC in the simulation. This is because of the assumption that no collision happens in HCC. (The collision resolution is hidden by assuming a fixed probability $p_{s}$ to successfully gain access to the channel.) In HCC, all users contend on the same channel, whereas, in MRCC, this contention is spread across channels. As a result, during the control phase, MRCC can harm the PU with a higher probability, i.e., up to $M_{D} p_{s}\left(1-p_{d}\right)$ (assuming a contention on every channel), while HCC only interferes with probability $p_{s}\left(1-p_{d}\right)$. In reality, the interference should, however, happen with the same probability. Finally, in Fig. 9(b), we note that, again, SPCC, MRCC, and HCC have a lower interference when the on and off durations of the PU are longer. For DCC, the interference does not vary as a function of the PU traffic patterns.

\section{CONCLUSION}

In this paper, a very detailed performance study of OSA MAC protocols has been presented. Using an analytical model and detailed simulations, we were able to assess different OSA MAC designs in a cohesive manner. This is the first study that performs such a detailed assessment since the CC design in OSA context is often neglected.

We draw important conclusions from our study. First, we show that the negative impact of PU activity on the CC can be mitigated by transmitting long SU packets by decreasing the number of channels used. Next, although the scanning length and capture effects have equal impacts on all considered OSA MAC classes, a proper insight to these effects in the OSA context has been obtained. As a result, it is shown that the impact of capture on the OSA network throughput is small when the PU detector is properly designed. The impact of the scanning configuration was, however, large, and there is a single optimal point of operation. All OSA MAC classes linearly scale with the increase in PU activity, but MRCC achieves the highest throughput since it can more efficiently use the available white spaces. Interestingly, the throughput achieved by MRCC is higher when the PU activity is organized in longer on and off periods. Delay steeply increases with PU activity when the network becomes congested for all OSA MACs. Again, MRCC achieves the lowest delay. Furthermore, MRCC delay is also not significantly affected by the PU packet lengths (on and off periods). We conclude that, from the OSA network perspective, MRCC is the best OSA MAC protocol since it achieves the highest throughput and lowest delay for any PU activity pattern while causing a low interference to the PU (particularly when the PU has long on/off times). MRCC is the most efficient in finding and using the random white spaces. Moreover, its implementation complexity is comparable to the other multichannel MAC classes. Major conclusions are also summarized in Table $\mathrm{V}$.

We have shown that OSA MACs that spread requests among all possible channels, e.g., MRCC, perform best among all the proposed OSA MAC classes. However, the hopping sequence
TABLE V

Main Results. (VL: Very Low, L: Low, M: Moderate, H: High)

\begin{tabular}{r|c|c|c|c}
\hline & DCC & HCC & SPCC & MRCC \\
\hline \hline PU Channels Scalability & $\mathrm{L}$ & $\mathrm{L}$ & $\mathrm{VL}$ & $\mathrm{H}$ \\
\hline Impact of Average PU Activity & $\mathrm{M}$ & $\mathrm{M}$ & $\mathrm{H}$ & $\mathrm{L}$ \\
\hline Impact of PU Activity Duration & $\mathrm{H}$ & $\mathrm{L}$ & $\mathrm{H}$ & $\mathrm{L}$ \\
\hline Induced PU Interference & $\mathrm{H}$ & $\mathrm{M}$ & $\mathrm{M}$ & $\mathrm{M}$ \\
\hline Impact of scanning & \multicolumn{5}{|c}{$\mathrm{M}$ (if properly designed) } \\
\hline Impact of packet capture & \multicolumn{5}{|c}{ (if properly designed) }
\end{tabular}

of MRCC implemented in the simulator is not adaptable to the level of activity of the PUs on each of the channels since we have assumed that all channels have been utilized in the same way by the PUs. In the case of nonuniform channel utilization, as future work, our plan is to extend MRCC with adaptability mechanisms.

\section{ACKNOWLEDGMENT}

The authors would like to thank the anonymous reviewers for their very insightful comments and Prof. Y. Narahari from the Indian Institute of Science, Bangalore, for valuable suggestions on the optimization part of this paper.

\section{REFERENCES}

[1] P. Pawełczak, S. Pollin, H.-S. W. So, A. Bahai, R. V. Prasad, and R. Hekmat, "Quality of service assessment of opportunistic spectrum access: A medium access control approach," IEEE Wireless Commun., vol. 15, no. 5, pp. 20-29, Oct. 2008.

[2] Q. Zhao and B. M. Sadler, "A survey of dynamic spectrum access: Signal processing, networking, and regulatory policy," IEEE Signal Process. Mag., vol. 24, no. 3, pp. 79-89, May 2007.

[3] R. V. Prasad, P. Pawełczak, J. Hoffmeyer, and S. Berger, "Cognitive functionality in next generation wireless networks: Standardization efforts," IEEE Commun. Mag., vol. 46, no. 4, pp. 72-78, Apr. 2008.

[4] S. Pollin, "Coexistence and dynamic sharing in cognitive radio networks," in Cognitive Wireless Communication Networks, E. Hossain and V. K. Bhargava, Eds. New York: Springer-Verlag, 2007.

[5] D. Raychaudhuri and X. Jing, "A spectrum etiquette protocol for efficient coordination of radio devices in unlicensed bands," in Proc. IEEE PIMRC, Beijing, China, Sep. 7-10, 2003, pp. 172-176.

[6] J. Pérez-Romero, O. Sallent, R. Agustí, and L. Giupponi, "A novel ondemand cognitive pilot channel enabling dynamic spectrum allocation," in Proc. IEEE DySPAN, Dublin, Ireland, Apr. 17-20, 2007, pp. 46-54.

[7] M. M. Buddhikot, P. Kolodzy, S. Miller, K. Ryan, and J. Evans, "DIMSUMNet: New directions in wireless networking using coordinated dynamic spectrum access," in Proc. IEEE WoWMoM, Taormina, Italy, 2005, pp. 78-85.

[8] C.-T. Chou, S. Shankar N, H. Kim, and K. G. Shin, "What and how much to gain by spectrum agility?" IEEE J. Sel. Areas Commun., vol. 25, no. 3 , pp. 576-588, Apr. 2007.

[9] Y. Xing, R. Chandramouli, S. Mangold, and S. Shankar N, "Dynamic spectrum access in open spectrum wireless networks," IEEE J. Sel. Areas Commun., vol. 24, no. 3, pp. 626-637, Mar. 2006.

[10] J. Mo, H.-S. W. So, and J. Walrand, "Comparison of multi-channel MAC protocols," IEEE Trans. Mobile Comput., vol. 7, no. 1, pp. 50-65, Jan. 2008.

[11] S. Sengupta, S. Brahma, M. Chatterjee, and S. Shankar N, "Enhancements to cognitive radio based IEEE 802.22 air-interface," in Proc. IEEE ICC, Glasgow, U.K., Jun. 24-28, 2007, pp. 5155-5160.

[12] P. Pawełczak, S. Pollin, H.-S. W. So, A. Motamedi, A. Bahai, R. V. Prasad, and R. Hekmat, "State of the art in opportunistic spectrum access MAC design: Research challenges," in Proc. ICST/IEEE CrownCom, Singapore, May 15-17, 2008.

[13] L. Ma, X. Han, and C.-C. Shen, "Dynamic open spectrum sharing MAC protocol for wireless ad hoc networks," in Proc. IEEE DySPAN, Baltimore, MA, Nov. 8-11, 2005, pp. 203-213.

[14] H. Su and X. Zhang, "Cross-layer based opportunistic MAC protocols for QoS provisioning over cognitive radio wireless networks," IEEE J. Sel. Areas Commun., vol. 26, no. 1, pp. 118-129, Jan. 2008. 
[15] A. Motamedi and A. Bahai, "MAC protocol design for spectrum-agile wireless networks: Stochastic control approach," in Proc. IEEE DySPAN, Dublin, Ireland, Apr. 17-20, 2007, pp. 448-451.

[16] A. C.-C. Hsu, D. S. L. Wei, and C.-C. J. Kuo, "A cognitive MAC protocol using statistical channel allocation for wireless ad-hoc networks," in Proc. IEEE WCNC, Hong Kong, Mar. 11-15, 2007, pp. 105-110.

[17] H. Nan, T.-I. Hyon, and S.-J. Yoo, "Distributed coordinated spectrum sharing MAC protocol for cognitive radio," in Proc. IEEE DySPAN, Dublin, Ireland, Apr. 17-20, 2007, pp. 240-249.

[18] C. Cordeiro and K. Challapali, "C-MAC: A cognitive MAC protocol for multi-channel wireless networks," in Proc. IEEE DySPAN, Dublin, Ireland, Apr. 17-20, 2007, pp. 147-157.

[19] S. Sankaranarayanan, P. Papadimitratos, and A. Mishra, "A bandwidth sharing approach to improve licensed spectrum utilization," IEEE Commun. Mag., vol. 43, no. 12, pp. S10-S14, Dec. 2005.

[20] X. Liu and Z. Ding, "ESCAPE: A channel evacuation protocol for spectrum-agile networks," in Proc. IEEE DySPAN, Dublin, Ireland, Apr. 17-20, 2007, pp. 292-302.

[21] M. Timmers, A. Dejonghe, L. Van der Perre, and F. Catthoor, "A distributed multichannel MAC protocol for cognitive radio networks with primary user recognition," in Proc. ICST/IEEE CrownCom, Orlando, FL, Aug. 1-3, 2007, pp. 216-223.

[22] J. Zhao, H. Zheng, and G.-H. Yang, "Distributed coordination in dynamic spectrum allocation networks," in Proc. IEEE DySPAN, Baltimore, MA, Nov. 8-11, 2005, pp. 259-268.

[23] J. Jia, Q. Zhang, and X. Shen, "HC-MAC: A hardware-constrained cognitive MAC for efficient spectrum management," IEEE J. Sel. Areas Commun., vol. 26, no. 1, pp. 106-117, Jan. 2008.

[24] N. Choi, M. Patel, and S. Venkatesan, "A full duplex multichannel MAC protocol for multi-hop cognitive radio networks," in Proc. ICST/IEEE CrownCom, Mykonos Island, Greece, Jun. 8-10, 2006.

[25] T. Shu, S. Cui, and M. Krunz, "Medium access control for multichannel parallel transmission in cognitive radio networks," in Proc. IEEE GLOBECOM, San Francisco, CA, Nov. 27-Dec. 1, 2006.

[26] G. Auer, H. Haas, and P. Omiyi, "Interference aware medium access for dynamic spectrum sharing," in Proc. IEEE DySPAN, Dublin, Ireland, Apr. 17-20, 2007, pp. 399-402.

[27] B. Hamdaoui and K. G. Shin, "OS-MAC: An efficient MAC protocol for spectrum-agile wireless networks," IEEE Trans. Mobile Comput., vol. 7, no. 8, pp. 915-930, Aug. 2008.

[28] Q. Zhao, L. Tong, A. Swami, and Y. Chen, "Decentralized cognitive MAC for opportunistic spectrum access in ad hoc networks: A POMDP framework," IEEE J. Sel. Areas Commun., vol. 25, no. 3, pp. 589-600, Apr. 2007

[29] L. Ma, C.-C. Shen, and B. Ryu, "Single-radio adaptive channel algorithm for spectrum agile wireless ad hoc networks," in Proc. IEEE DySPAN, Dublin, Ireland, Apr. 17-20, 2007, pp. 547-558.

[30] Y. Chen, Q. Zhao, and A. Swami, "Distributed cognitive MAC for energyconstrained opportunistic spectrum access," in Proc. IEEE MILCOM, Washington, DC, Oct. 23-25, 2006, pp. 1-7.

[31] H.-S. W. So, J. Walrand, and J. Mo, "McMAC: A multichannel MAC proposal for ad-hoc wireless networks," in Proc. IEEE WCNC, Hong Kong, Mar. 11-15, 2007, pp. 334-339.

[32] T. Yucek and H. Arslan, "A survey of spectrum sensing algorithms for cognitive radio applications," IEEE Commun. Surveys Tuts., Apr. 2007, to be published.

[33] F. F. Digham, M.-S. Alouini, and M. K. Simon, "On the energy detection of unknown signals over fading channels," in Proc. IEEE ICC, Anchorage, AK, May 11-15, 2003, pp. 3575-3579.

[34] P. Pawełczak, R. V. Prasad, and R. Hekmat, "Opportunistic spectrum multichannel OFDMA," in Proc. IEEE ICC, Glasgow, U.K., Jun. 24-28, 2007, pp. 5439-5444.

[35] J. C. Arnbak and W. van Blitterswijk, "Capacity of slotted ALOHA in Rayleigh-fading channels," IEEE J. Sel. Areas Commun., vol. SAC-5, no. 2, pp. 261-269, Feb. 1987.

[36] P. Pawełczak, S. Pollin, H.-S. W. So, A. Bahai, R. V. Prasad, and R. Hekmat, "Comparison of opportunistic spectrum multichannel medium access control protocols," in Proc. IEEE GLOBECOM, New Orleans, LA, Nov. 30-Dec. 4, 2008, pp. 1-6.

[37] A. Birman, "Computing approximate blocking probabilities for a class of all-optical networks," IEEE J. Sel. Areas Commun., vol. 14, no. 5, pp. 852-857, Jun. 1996.

[38] S. Kameshwaran and Y. Narahari, "Efficient algorithms for nonconvex piecewise linear knapsack problems," Eur. J. Oper. Res., vol. 192, no. 1, pp. 56-68, Jan. 2009.

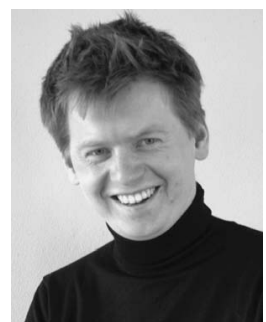

Przemysław Pawełczak (S'01) received the M.Sc. (Hons.) degree in electronics and telecommunications engineering from Wrocław University of Technology, Wrocław, Poland, in 2004. He is currently working toward the Ph.D. degree in opportunistic spectrum access networks with the Department of Electrical Engineering, Mathematics, and Computer Science, Delft University of Technology, Delft, The Netherlands.

From 2004 to 2005, he was a Staff Member with the Siemens Software Development Center, Wrocław. During Fall 2007, he was a Visiting Scholar with the Connectivity Laboratory, University of California, Berkeley. His research interests include performance analysis and medium access control protocol design for wireless networks.

Mr. Pawełczak is a member of the IEEE Communications Society and a Voting Member of the IEEE SCC41 Standardization Committee. He has been a Cooriginator and an Organizing Committee Member of the Cognitive Radio workshops collocated with the IEEE International Conference on Communications in 2007, 2008, and 2009. He was the recipient of the Annual KIVI NIRIA Telecom Prize for Best Ph.D. Student in Telecommunications in The Netherlands in 2008 .

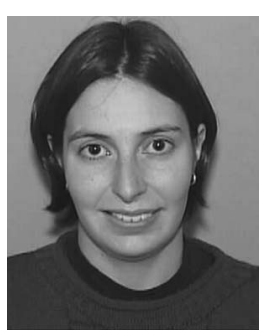

Sofie Pollin (M'06) received the M.Sc. degree in electrical engineering and the Ph.D. degree (with honors) from the Katholieke Universiteit, Leuven, Belgium, in 2002 and 2006, respectively.

Since October 2002, she has been a Researcher with the Wireless Research Group, Interuniversity Microelectronics Center (IMEC), Leuven. In the summer of 2004, she was a Visiting Scholar with National Semiconductor, Santa Clara, CA. In the summer of 2005, she was a Visitor with the University of California, Berkeley, where she is currently a Postdoctoral Researcher with the Department of Electrical Engineering and Computer Science, working on coexistence issues in wireless communication networks.

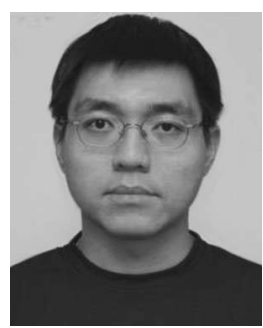

Hoi-Sheung Wilson So (M'08) received the B.S. degree from Cornell University, Ithaca, NY, in 1997 and the M.Sc. and Ph.D. degrees from the University of California, Berkeley, in 2000 and 2006, respectively, all in computer science.

$\mathrm{He}$ is currently with the Siemens Technologyto-Business Center, Berkeley. His current research interests include wireless protocol design and multichannel media access control protocols for wireless networks.

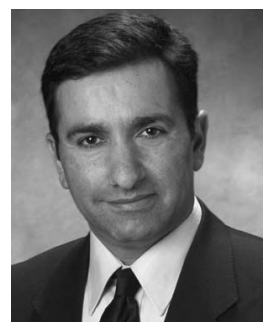

Ahmad R. S. Bahai (M'95) received the M.Sc. degree in electrical engineering from Imperial College, University of London, London, U.K., in 1988 and the Ph.D. degree from the University of California, Berkeley, in 1993.

He was the Technical Manager of the Advance Wireless Technology Group, AT\&T Bell Laboratories, until 1997. He is currently a Professorin-Residence with the Department of Electrical Engineering and Computer Science, University of California; a Consulting Professor with the Department of Electrical Engineering, Stanford University, Stanford, CA; and Executive Advisor to National Semiconductor, Santa Clara, CA. He has served as a Fellow and Chief Technical Officer of National Semiconductor for five years. $\mathrm{He}$ is the author of the first textbook on OFDM entitled Multi-Carrier Digital Communications (Springer, 2004). He coinvented the multicarrier spread spectrum theory, which is being used in most modern wireless systems and standards. His research interest includes adaptive/mixed signal processing and communication system design.

Dr. Bahai has been an Associate Editor for the IEEE COMMUNICATIONS LETTERS for five years. 


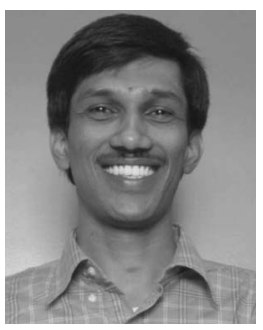

R. Venkatesha Prasad (M'95) received the B.Sc. degree in electronics and communication engineering and the M.Tech. degree in industrial electronics from the University of Mysore, Mysore, India, in 1991 and 1994, respectively, and the Ph.D. degree from Indian Institute of Science (IISc) Bangalore, India, in 2003.

In 1994 and 1996, he was a Consultant and Project Associate for ERNET Laboratory of Electronics and Communication Engineering, IISc. From 1999 to 2003, he was a Consultant for CEDT, IISc, for VoIP application developments, as part of the Nortel-Networks-sponsored project. From 2003 to 2005, he headed a team of engineers at the Esqube Communication Solutions Pvt. Ltd. for the development of various real-time networking applications. Since 2005, he has been with the Wireless and Mobile Communications Group, Department of Electrical Engineering, Mathematics and Computer Science, Delft University of Technology, Delft, The Netherlands, working on the European-Union-funded projects MAGNET/MAGNET Beyond and PNP 2008, and guiding students. He is also a Consultant for Esqube on a part-time basis.

Dr. Prasad is a member of the IEEE SCC41 Standardization Committee. He has been a Coordinator and a Technical Program Committee (TPC) member of CogNet, Pernets, and many other workshops. He has been a Reviewer and a TPC member of many conferences and has chaired some of the committees.

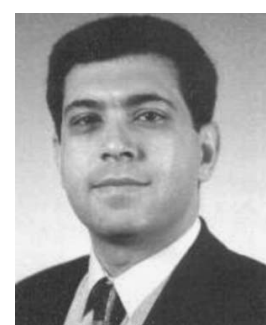

Ramin Hekmat received the M.Sc. degree in electrical engineering and the Ph.D. degree, for his work related to ad hoc networks, from Delft University of Technology (TU Delft), Delft, The Netherlands, in 1990 and 2005, respectively.

Since then, he has been with several telecommunication companies in The Netherlands and the United States, working in research and development and holding managerial positions. He is currently an Associate Professor with the Department of Electrical Engineering, Mathematics, and Computer Science, TU Delft, as well as a Strategic Consultant with Capgemini Nederland B.V., Utrecht, The Netherlands. His research interests include multiuser communication systems, wireless communications, and peer-to-peer networks. 